\title{
New heavenly double copies
}

\author{
Erick Chacón, ${ }^{a}$ Hugo García-Compeán, ${ }^{b}$ Andrés Luna, ${ }^{c}$ Ricardo Monteiro ${ }^{a}$ and \\ Chris D. White ${ }^{a}$ \\ ${ }^{a}$ Centre for Research in String Theory, School of Physics and Astronomy, \\ Queen Mary University of London, \\ 327 Mile End Road, London E1 4NS, U.K. \\ ${ }^{b}$ Departamento de Física, Centro de Investigación y de Estudios Avanzados del \\ Instituto Politécnico Nacional, \\ P.O. Box 14-740, CP. 07000, México D.F., Mexico \\ ${ }^{c}$ Mani L. Bhaumik Institute for Theoretical Physics, Department of Physics and Astronomy, \\ University of California, \\ Los Angeles, CA 90095, U.S.A. \\ E-mail: e.c.chaconramirez@qmul.ac.uk, compean@fis.cinvestav.mx, \\ luna@physics.ucla.edu, ricardo.monteiro@qmul.ac.uk, \\ christopher.white@qmul.ac.uk
}

ABSTRACT: The double copy relates scattering amplitudes and classical solutions in YangMills theory, gravity, and related field theories. Previous work has shown that this has an explicit realisation in self-dual YM theory, where the equation of motion can be written in a form that maps directly to Plebański's heavenly equation for self-dual gravity. The selfdual YM equation involves an area-preserving diffeomorphism algebra, two copies of which appear in the heavenly equation. In this paper, we show that this construction is a special case of a wider family of heavenly-type examples, by (i) performing Moyal deformations, and (ii) replacing the area-preserving diffeomorphisms with a less restricted algebra. As a result, we obtain a double-copy interpretation for hyper-Hermitian manifolds, extending the previously known hyper-Kähler case. We also introduce a double-Moyal deformation of the heavenly equation. The examples where the construction of Lax pairs is possible are manifestly consistent with Ward's conjecture, and suggest that the classical integrability of the gravity-type theory may be guaranteed in general by the integrability of at least one of two gauge-theory-type single copies.

KEywords: Classical Theories of Gravity, Integrable Field Theories, Integrable Hierarchies, Non-Commutative Geometry

ArXiv EPrint: 2008.09603 


\section{Contents}

1 Introduction 1

2 The self-dual double copy 3

2.1 Review 3

2.2 Lax pairs 7

2.3 Infinite tower of conserved currents 8

3 Moyal deformations in gauge theory and gravity 9

$\begin{array}{ll}3.1 \text { Deformed self-dual gravity } & 12\end{array}$

3.1.1 Deformed self-dual gravity as undeformed self-dual Yang-Mills $\quad 14$

$\begin{array}{ll}3.2 & \text { Deformed self-dual Yang-Mills } \\ & 16\end{array}$

$\begin{array}{lll}3.3 & \text { Doubly deformed self-dual gravity } & 18\end{array}$

4 Generalising to the diffeomorphism algebra 20

4.1 Hyper-Hermitian manifold 21

4.2 Other Diff theories 23

$5 \quad$ Summary of results $\quad 23$

6 Conclusions 25

\section{Introduction}

A major focus of modern theoretical physics is the remarkable web of connections between different field theories of interest. In this paper, we explore one such connection, the socalled double copy between gauge theory and gravity, which has been the basis of much work over the past decade due to its applications. The double copy originated in the study of perturbative scattering amplitudes, first in string theory [1] and later in quantum field theory and gravity [2-4], where it has been studied at various loop orders, both with and without supersymmetry. More recently, the notion of double-copy relations between theories has been extended to the context of the classical limit of the theories using a variety of approaches. These include relating exact, algebraically special solutions to the equations of motion of the theories [5-35], as well as applications to perturbative methods. Some examples of the latter are constructing perturbative metrics directly [36], solving the equations of motion in a world-line formalism [37-46], relating linearised solutions using a convolution approach [47-57], as well as applying double-copy ideas to the computation of quantities of interest to gravitational wave astronomy, mainly in the post-Minkowskian regime [58-71]. Closer to the idea of this paper, there is a large body of literature on 
webs of theories related by the double copy, e.g., [72-80]. A comprehensive review of these developments may be found in refs. [81, 82].

A crucial idea, which fostered the development of the double copy, is the colourkinematics duality of Bern, Carrasco and Johansson (BCJ) [2]. This 'duality' remains a conjecture at loop level, but is well established for tree-level (i.e., classical) gauge theory. It states that the scattering amplitudes can be written in such a way that (apart from scalartype propagators) the kinematic dependence has the same algebraic properties as the colour dependence, thus hinting that there is a kinematic analogue of the colour Lie algebra in gauge theory. In particular, there is a kinematic analogue for the Jacobi identity of the colour Lie algebra, which gives rise to a notion of kinematic algebra. Upon taking the double copy, the colour information is replaced by another copy of its kinematic analogue, so that two kinematic algebras appear in gravity scattering amplitudes. In fact, starting from two distinct gauge theories (e.g., degree of supersymmetry or self-duality constraint), the two kinematic algebras in the gravity theory, each extracted from one of the 'single copies', will be distinct. Despite some progress, the mathematical interpretation of a kinematic algebra remains elusive, partly because there is no understanding of the colour-kinematics duality at the level of the action or the equations of motion. This would rely on a formulation of gauge theory with a single cubic interaction vertex, whereas the usual Yang-Mills action has also a four-point vertex. This cubic vertex would have as colour factor the usual Lie algebra structure constant, $f^{a b c}$, and as kinematic factor the putative structure constant of a kinematic algebra.

There is an exception, however, where the kinematic algebra has been fully understood. Reference [83] circumvented the difficulties of the general problem by restricting to the selfdual sector of pure Yang-Mills theory. In a long-known formulation [84], the action of selfdual YM involves a single adjoint-valued degree of freedom, and is manifestly cubic. It is then possible to completely elucidate the kinematic algebra alluded to above, which turns out to correspond to certain area-preserving diffeomorphisms (n.b. in the mathematical literature, this group is often referred to as SDiff or $\mathrm{SDiff}_{2}$, and the algebra as sdiff or sdiff $_{2}$ ). Furthermore, the equations of motion can be written in terms of an interaction involving the product of two sets of structure constants, for the colour and kinematic Lie algebras respectively. Thus, the colour-kinematics duality becomes explicit, and the double copy to self-dual gravity straightforward. The equation of motion for self-dual gravity arises naturally in the form of Plebański's (second) heavenly equation [85].

There have been many interesting results regarding kinematic algebras. They have been explored via algorithms for obtaining colour-kinematics-dual expressions for scattering amplitudes, e.g., in [86-96], and via a variety of algebraic and geometric approaches, e.g., in [97-101]. And yet, to date, it has not been possible to generalise the very explicit construction for the self-dual sectors to the full Yang-Mills theory and gravity case. This motivates looking for examples of kinematic algebras that go beyond those of ref. [83]. That is the main aim of our paper, and we will seek to generalise the results of ref. [83] in two key ways. Firstly, we may form qualitatively different types of gauge and gravity theories by replacing the Lie and Poisson brackets appearing in the former and latter with their Moyal deformations [102]. These arise in alternative formulations of quantum 
mechanics $[103,104]$ as well as in the study of field theories in non-commutative spacetimes; see e.g. refs. $[105,106]$ for pedagogical reviews. The Moyal bracket constitutes the most general bracket one can write down for a Lie algebra of functions of two variables [107], here corresponding to the two-dimensional space on which the diffeomorphisms act. Secondly, we will consider replacing the sdiff algebra discussed above with the set of arbitrary rather than area-preserving - two-dimensional diffeomorphisms (diff). Both of the above generalisations lead to new instances of the double copy, in which the colour-kinematics duality is manifest. These results are summarised in the table of theories of section 5 .

A second motivation for our paper is to explore the classical integrability (or otherwise) of the gauge and gravity theories related by the double copy, an aspect that was not considered in ref. [83]. As is well-known, both self-dual Yang-Mills theory and self-dual gravity are integrable, and thus admit an infinite number of conserved charges. This integrability can be expressed in terms of a Lax pair, and we will find a hitherto unexplored double copy interpretation of Lax pairs in different theories. This suggests that integrability of a gravity theory is "inherited" from its corresponding gauge theories via the double copy. Also, in considering the more general theories outlined above, we will find that integrability in the gravity theory can be obtained even if only one of the gauge theories in the double copy is integrable.

The structure of our paper is as follows. In section 2, we review the self-dual double copy, and relate this to ideas regarding integrability. We consider Moyal deformations in section 3 . In section 4 , we look at generalising the algebra of diffeomorphisms in the gauge theory, and construct a corresponding gravity theory. We summarise the web of theories studied in this paper in section 5, and the general form of the different Lax pairs and the infinite tower of linearised symmetries is examined as well. Finally, we discuss our results and conclude in section 6 .

\section{The self-dual double copy}

\subsection{Review}

In this section, we review salient details of the double copy in the self-dual sector described in ref. [83]. This will set up useful notation for what follows, whilst also allowing us to draw attention to aspects of integrability, which were not examined in [83]. Our starting point is to consider pure Yang-Mills theory. The (vacuum) equation of motion is given by

$$
D^{\mu} F_{\mu \nu}=0
$$

where

$$
F_{\mu \nu}=\partial_{\mu} A_{\nu}-\partial_{\nu} A_{\mu}-i g\left[A_{\mu}, A_{\nu}\right]
$$

is the field strength tensor in terms of the gauge field $A_{\mu} \equiv A_{\mu}^{a} T^{a}$, and $T^{a}$ is a generator of the Lie algebra $\mathbf{g}$ of the gauge group $G$. Also, $D_{\mu}=\partial_{\mu}-i g A_{\mu}$ is the covariant derivative, where the gauge field in the second term acts in the adjoint representation. The equations of motion take a particularly simple form if one considers self-dual solutions, for which the field strength tensor satisfies

$$
F_{\mu \nu}=\frac{i}{2} \epsilon_{\mu \nu \rho \sigma} F^{\rho \sigma}
$$


One may introduce coordinates

$$
u=t-z, \quad v=t+z, \quad w=x+i y, \quad \bar{w}=x-i y
$$

such that the Minkowski line element becomes

$$
d s^{2}=d u d v-d w d \bar{w}
$$

and the self-duality conditions can be written as

$$
\begin{aligned}
F_{u w} & =0, \\
F_{v \bar{w}} & =0, \\
F_{u v}-F_{w \bar{w}} & =0 .
\end{aligned}
$$

From eq. (2.6) and a light-cone gauge choice, we may take

$$
A_{u}=A_{w}=0 .
$$

It follows from eq. (2.8) that there must exist an adjoint-valued scalar function $\Phi \equiv \Phi^{a} T^{a}$ such that

$$
A_{v}=-\partial_{w} \Phi, \quad A_{\bar{w}}=-\partial_{u} \Phi .
$$

Physically, $\Phi$ represents the single polarisation state that remains in the gauge field upon projecting to the self-dual sector, and eq. (2.7) implies that it satisfies the following equation of motion:

$$
\partial^{2} \Phi+i g\left[\partial_{w} \Phi, \partial_{u} \Phi\right]=0,
$$

where $\partial^{2} \equiv \partial_{u} \partial_{v}-\partial_{w} \partial_{\bar{w}}$. We follow ref. [83], to rewrite this as an integral equation in momentum space. Fourier transforming eq. (2.11) and rearranging yields

$$
\begin{aligned}
\Phi(k) & =-i g \int d^{D} x e^{-i x \cdot\left(p_{1}+p_{2}-k\right)} \int d p_{1} \int d p_{2} \frac{1}{k^{2}}\left(p_{1 w} p_{2 u}-p_{1 u} p_{2 w}\right) \Phi\left(p_{1}\right) \Phi\left(p_{2}\right) \\
& =-i g \int d p_{1} d p_{2} \frac{\delta\left(p_{1}+p_{2}-k\right)}{k^{2}} X\left(p_{1}, p_{2}\right) \Phi\left(p_{1}\right) \Phi\left(p_{2}\right),
\end{aligned}
$$

where we have employed the shorthand notation

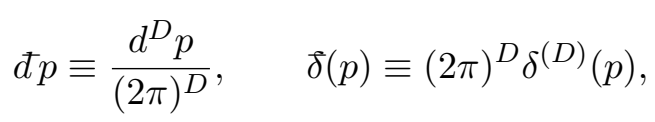

and, in the second line, have defined the kinematic structure constant

$$
X\left(p_{1}, p_{2}\right)=p_{1 w} p_{2 u}-p_{1 u} p_{2 w}
$$

Antisymmetry of the latter under $p_{1} \leftrightarrow p_{2}$ means that one may replace the product of scalar fields with a commutator, to finally write

$$
\Phi^{a}(k)=\frac{g}{2} \int d p_{1} d p_{2} \frac{\delta\left(p_{1}+p_{2}-k\right)}{k^{2}} X\left(p_{1}, p_{2}\right) f^{a b c} \Phi^{b}\left(p_{1}\right) \Phi^{c}\left(p_{2}\right) .
$$


The $f^{a b c}$ are the structure constants of the Lie algebra $\mathbf{g},\left[T^{a}, T^{b}\right]=i f^{a b c} T^{c}$. The kinematic objects in eq. (2.14) are also the structure constants of a Lie algebra, the Jacobi identity being

$$
X\left(p_{1}, p_{2}\right) X\left(p_{1}+p_{2}, p_{3}\right)+X\left(p_{2}, p_{3}\right) X\left(p_{2}+p_{3}, p_{1}\right)+X\left(p_{3}, p_{1}\right) X\left(p_{3}+p_{1}, p_{2}\right)=0 .
$$

The kinematic Lie algebra is infinitely dimensional, with generators of the form

$$
L_{k}=e^{-i k \cdot x}\left(-k_{w} \partial_{u}+k_{u} \partial_{w}\right)
$$

satisfying

$$
\left[L_{p_{1}}, L_{p_{2}}\right]=i X\left(p_{1}, p_{2}\right) L_{p_{1}+p_{2}} .
$$

It is the Lie algebra sdiff of area-preserving diffeomorphisms in the $(w, u)$ plane. We can also interpret it as a Poisson algebra, with

$$
\left\{e^{-i p_{1} x}, e^{-i p_{2} x}\right\}=-X\left(p_{1}, p_{2}\right) e^{-i\left(p_{1}+p_{2}\right) x},
$$

where the Poisson bracket is

$$
\{A, B\}=\partial_{w} A \partial_{u} B-\partial_{u} A \partial_{w} B
$$

We see that the self-dual YM equation (2.15) is precisely that expected from a Lagrangian involving a cubic interaction only, whose Feynman rule involves the product of structure constants for two Lie algebras, corresponding respectively to colour, $f^{a b c}$, and kinematics, $X(p, q)$. This makes manifest the BCJ duality between colour and kinematics of ref. [2], in a sector where the kinematic algebra is a straightforward Lie algebra.

Given eq. (2.15) and the interpretation given above, one may replace the colour structure constants with a second set of kinematic structure constants, i.e.

$$
f^{a b c} \rightarrow X\left(p_{1}, p_{2}\right)
$$

obtaining the momentum-space equation of motion

$$
\phi(k)=\frac{\kappa}{2} \int đ p_{1} đ p_{2} \frac{\delta\left(p_{1}+p_{2}-k\right)}{k^{2}} X\left(p_{1}, p_{2}\right)^{2} \phi\left(p_{1}\right) \phi\left(p_{2}\right),
$$

for a scalar field $\phi$. We now identify the coupling constant as the gravitational coupling $\kappa=\sqrt{32 \pi G_{N}}$, in terms of Newton's constant $G_{N}$. In coordinate space, eq. (2.22) is simply the Plebański equation for self-dual gravity, also known as the (second) heavenly equation:

$$
\partial^{2} \phi+\kappa\left\{\partial_{w} \phi, \partial_{u} \phi\right\}=0 .
$$

Thus, the double copy is particularly clear, and amounts to simply replacing the colour algebra with its kinematic counterpart. One can also go the other way in eq. (2.21), replacing the area-preserving diffeomorphisms Lie algebra with a second 'colour' Lie algebra $\tilde{\mathrm{g}}$ associated to a Lie group $\tilde{G}$. In this case, one obtains solutions of the so-called biadjoint scalar field theory, whose equation of motion is

$$
\partial^{2} \Phi^{a a^{\prime}}+y f^{a b c} \tilde{f}^{a^{\prime} b^{\prime} c^{\prime}} \Phi^{b b^{\prime}} \Phi^{c c^{\prime}}=0
$$




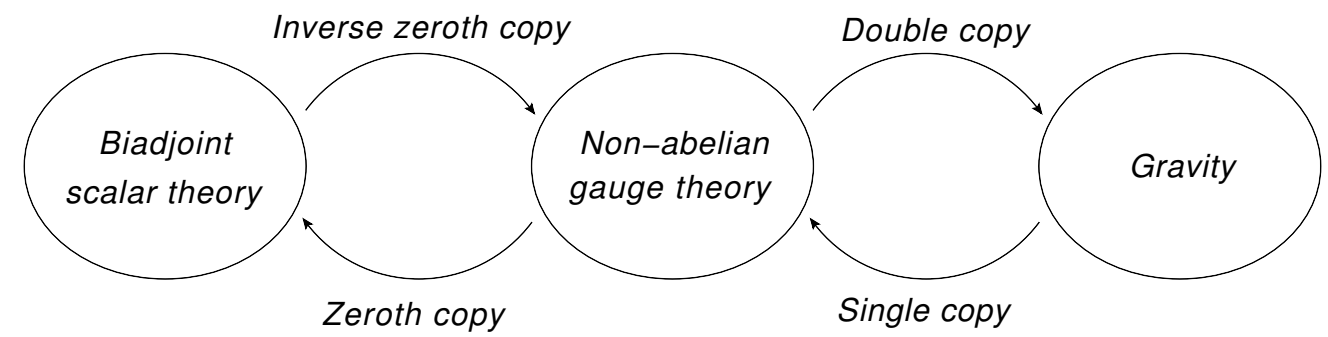

Figure 1. Various field theories and the relationships between them. Figure taken from ref. [21].

or, in momentum space,

$$
\Phi^{a a^{\prime}}(k)=y \int đ p_{1} \tau p_{2} \frac{\delta\left(p_{1}+p_{2}-k\right)}{k^{2}} f^{a b c} \tilde{f}^{a^{\prime} b^{\prime} c^{\prime}} \Phi^{b b^{\prime}}\left(p_{1}\right) \Phi^{c c^{\prime}}\left(p_{2}\right) .
$$

Here, $y$ is a coupling constant, and $f^{a b c}, \tilde{f}^{a^{\prime} b^{\prime} c^{\prime}}$ are structure constants for the two Lie groups. There is thus a hierarchy of theories as shown in figure 1, which in the self-dual sector involves scalar field theories with a cubic interaction. In moving to the right in the figure, one removes an adjoint index from the field, and replaces a set of colour structure constants in the field equation with a set of kinematic structure constants. The biadjoint scalar theory plays a crucial role in the formulas expressing the double copy of scattering amplitudes. From now on, we set the different coupling constants $(y, g, \kappa)$ to one.

This 'heavenly' example of the double copy is a different viewpoint to the well-known story in the integrability literature where self-dual gravity is recovered as a symmetry reduction of self-dual Yang-Mills theory, considering the gauge group as the area-preserving diffeomorphism group on the two-surface $\Sigma, \operatorname{SDiff}(\Sigma)$ [108-110]. This fact has been used to build hyper-Kähler metrics from solutions of two-dimensional reduced SDYM equations [111-113]. The double copy motivates the consideration of the biadjoint scalar theory mentioned above. Notice that self-dual Yang-Mills theory is a 'symmetry reduction' of the biadjoint scalar theory in the same way that self-dual gravity is a symmetry reduction of self-dual Yang-Mills theory.

Let us also comment on an alternative description of the self-dual theories. Whereas the second heavenly equation is eq. (2.23), the first heavenly equation reads

$$
\left\{\partial_{v} \Omega, \partial_{\bar{w}} \Omega\right\}=1,
$$

where $\Omega$ is the Kähler potential, and we use the $(w, u)$ Poisson bracket previously defined. The two heavenly equations are equivalent and arise due to distinct gauge choices [85]. A somewhat analogous formulation is possible in self-dual Yang-Mills, where instead of (2.10), the relevant equation is

$$
\left[\partial_{w} \Xi, \partial_{u} \Xi\right]=0,
$$

where $\Xi$ is Lie algebra-valued. This equation arises from a two-dimensional sigma model with pure $\mathrm{SU}(\mathrm{N})$ Wess-Zumino term, and is related to eq. (2.26) in the large-N limit [114], for reasons similar to those discussed in section 3.1.1. While equations (2.26) and (2.27) are closely related, the double copy structure is not at all clear, as in the case of $(2.23)$ 
and (2.11), which are based on a cubic interaction vertex, the preferred setting for making the double copy manifest. Still, it would be interesting to explore these and other alternative formulations, in the search for further insights into the double copy.

\subsection{Lax pairs}

There is an additional structure in the equations of motion (2.11) and (2.23) for the selfdual theories, that did not receive attention in ref. [83], but which will be useful to review for what follows. Namely, both self-dual Yang-Mills and gravity are known to be integrable theories, admitting an infinite number of conserved charges. The existence of a Lax pair indicates that a given theory may be integrable, and we will be interested in tracing how integrability is inherited by different theories in our double copy examples. For self-dual YM, this Lax pair consists of two operators obtained from the covariant derivative,

$$
L=D_{u}-\lambda D_{\bar{w}}, \quad M=D_{w}-\lambda D_{v},
$$

where $\lambda \in \mathbb{C} P^{1}$ is an arbitrary spectral parameter. It is then straightforward to show that the self-dual Yang-Mills equations of eqs. (2.6)-(2.8) follow from the compatibility condition

$$
[L, M]=0 .
$$

Note that for the explicit gauge choice of eqs. (2.9), (2.10), the above Lax pair can also be written as

$$
L=\partial_{u}-\lambda\left(\partial_{\bar{w}}+i \partial_{u} \Phi\right), \quad M=\partial_{w}-\lambda\left(\partial_{v}+i \partial_{w} \Phi\right) .
$$

The gravitational case can be expressed in a similar way. We can use known results to classify the types of manifolds that arise, based on the relevant Lax pair, as in $[115,116]$. More specifically, let us take $\mathbf{V}=\{U, V, W, \bar{W}\}$ to be four independent holomorphic vector fields on a four-dimensional complex manifold $\mathcal{M}$, such that

$$
L=U-\lambda \bar{W}, \quad M=W-\lambda V, \quad \lambda \in \mathbb{C} P^{1},
$$

are operators satisfying $\mathcal{L}_{L} v=\mathcal{L}_{M} v=0$ and eq. (2.29), for all $\lambda$, where $v$ is a non-zero holomorphic four-form and $\mathcal{L}$ denotes the Lie derivative. Then $\mathbf{V}$ forms a null tetrad for a so-called hyper-Kähler metric on $\mathcal{M}$. To make contact with the Plebański equation, we can choose coordinates such that

$$
\begin{array}{rlrl}
U & =\partial_{u}, \\
W & =\partial_{w}, & V & =\partial_{v}+\phi_{w w} \partial_{u}-\phi_{u w} \partial_{w}, \\
\bar{W} & =\partial_{\bar{w}}+\phi_{u w} \partial_{u}-\phi_{u u} \partial_{w},
\end{array}
$$

which corresponds to

$$
L=\partial_{u}-\lambda\left(\partial_{\bar{w}}+\phi_{u w} \partial_{u}-\phi_{u u} \partial_{w}\right), \quad M=\partial_{w}-\lambda\left(\partial_{v}+\phi_{w w} \partial_{u}-\phi_{u w} \partial_{w}\right),
$$

where we use the notation $\phi_{\mu \nu} \equiv \partial_{\mu} \partial_{\nu} \phi$. This Lax pair satisfies $[L, M]=0$ if $\phi$ satisfies the Plebański equation (2.23). Furthermore, by considering the holomorphic four-form

$$
v=d u \wedge d v \wedge d w \wedge d \bar{w}
$$


the Lax pair satisfies also the conditions $\mathcal{L}_{L} v=\mathcal{L}_{M} v=0$. Then, the vector fields in eq. (2.32) form a null tetrad for a hyper-Kähler metric.

The Lax pairs (2.30) and (2.33) are naturally related, as expected by the double copy. Let us introduce the Hamiltonian vector field in the $(u, w)$ plane, $\Phi_{f}$, defined by its action on functions, ${ }^{1}$

$$
\Phi_{f}(g) \equiv\{f, g\}
$$

so that

$$
\left[\Phi_{f}, \Phi_{g}\right]=\Phi_{\{f, g\}} \cdot
$$

Then, the Lax pair in self-dual gravity, in eq. (2.33), takes the form

$$
L=\partial_{u}-\lambda\left(\partial_{\bar{w}}+\partial_{u} \Phi_{\phi}\right), \quad M=\partial_{w}-\lambda\left(\partial_{v}+\partial_{w} \Phi_{\phi}\right),
$$

where the derivatives act only on the components of the Hamiltonian vector fields, that is, we define $\partial_{u} \Phi_{\theta_{n}} \equiv\left[\partial_{u}, \Phi_{\theta_{n}}\right]$ and $\partial_{w} \Phi_{\phi} \equiv\left[\partial_{w}, \Phi_{\phi}\right]$. This Lax pair mirrors the one in self-dual YM, eq. (2.30). In the self-dual gravity case, we can also write the Lax pair as

$$
L=\partial_{u}-\lambda\left(\partial_{\bar{w}}+\Phi_{\partial_{u} \phi}\right), \quad M=\partial_{w}-\lambda\left(\partial_{v}+\Phi_{\partial_{w} \phi}\right) .
$$

\subsection{Infinite tower of conserved currents}

Let us return to the question of integrability. With the Lax pairs in hand, it is possible to construct recursively (formally at least) an infinite number of symmetries, associated to the infinite-dimensional vector space of deformations of solutions to the equations of motion. This brief review is roughly based on the discussions in $[117,118]$. Let us take the self-dual YM example, with Lax pair given in (2.30). Notice that

$$
\left[\partial_{v}+i \partial_{w} \Phi, L \Psi\right]-\left[\partial_{\bar{w}}+i \partial_{u} \Phi, M \Psi\right]=\partial^{2} \Psi+i\left[\partial_{w} \Phi, \partial_{u} \Psi\right]+i\left[\partial_{w} \Psi, \partial_{u} \Phi\right]
$$

where we have used the Jacobi identity and the equation of motion (2.11). Hence, we obtain a solution $\Psi$ to the linearised equation of motion, $\Phi \mapsto \Phi+\Psi$, by solving the system

$$
L \Psi=0, \quad M \Psi=0 .
$$

The compatibility condition of this overdetermined system is $[L, M]=0$. Now, let us consider a solution to eq. (2.40) of the form

$$
\Psi(x, \lambda)=\sum_{n=0}^{\infty} \Psi_{n}(x) \lambda^{n},
$$

where $\lambda$ is the spectral parameter. Since

$$
\begin{gathered}
L \Psi=\sum_{n=0}^{\infty}\left(\partial_{u} \Psi_{n}-\left(\partial_{\bar{w}} \Psi_{n-1}+i\left[\partial_{u} \Phi, \Psi_{n-1}\right]\right)\right) \lambda^{n}, \\
M \Psi=\sum_{n=0}^{\infty}\left(\partial_{w} \Psi_{n}-\left(\partial_{v} \Psi_{n-1}+i\left[\partial_{w} \Phi, \Psi_{n-1}\right]\right)\right) \lambda^{n},
\end{gathered}
$$

\footnotetext{
${ }^{1}$ This Hamiltonian vector field is defined in the $(u, w)$ plane. However, the Hamiltonian functions depend on the coordinates $(u, v, w, \bar{w})$.
} 
with $\Psi_{-1} \equiv 0$, the system (2.40) is solved by an infinite tower of linearised solutions $\left\{\Psi_{n}\right\}$ constructed recursively via

$$
\partial_{u} \Psi_{n}=\partial_{\bar{w}} \Psi_{n-1}+i\left[\partial_{u} \Phi, \Psi_{n-1}\right], \quad \partial_{w} \Psi_{n}=\partial_{v} \Psi_{n-1}+i\left[\partial_{w} \Phi, \Psi_{n-1}\right]
$$

For instance, we can take $\Psi_{0}=\Psi_{0}^{a} T^{a}$, where $\Psi_{0}^{a}$ are constants, so that $\Psi_{1}=i\left[\Phi, \Psi_{0}^{a} T^{a}\right]$, etc. We can also express this hierarchy of infinitesimal symmetries in terms of a hierarchy of conserved currents, by considering

$$
J(x, \lambda)=\left(\partial_{w} \Psi\right) \partial_{u}-\left(\partial_{u} \Psi\right) \partial_{w}, \quad \text { such that }\left[\partial_{\mu}, J^{\mu}\right]=0 .
$$

The infinite tower of currents is given by the $\lambda$-expansion of $J^{\mu}$. Equivalently, we can state that, if (2.43) represents commuting flows of $\Psi_{n}$ along $u$ and $w$ (this is the compatibility condition $\left.\partial_{w u} \Psi_{n}=\partial_{u w} \Psi_{n}\right)$, then $\Psi_{n}$ is a linearised symmetry due to

$$
\partial^{2} \Psi_{n}+i\left[\partial_{w} \Phi, \partial_{u} \Psi_{n}\right]+i\left[\partial_{w} \Psi_{n}, \partial_{u} \Phi\right]=i\left[\partial^{2} \Phi+i\left[\partial_{w} \Phi, \partial_{u} \Phi\right], \Psi_{n-1}\right]=0
$$

where the last step follows from the equation of motion for $\Phi$. The first equality above relies on the Jacobi identity.

The discussion for self-dual gravity is analogous. For the Plebański equation, we can express the hierarchy of infinitesimal symmetries in terms of Hamiltonian vector fields $\left\{\Phi_{\theta_{n}}\right\}$ recursively via

$$
\partial_{u} \Phi_{\theta_{n}}=\partial_{\bar{w}} \Phi_{\theta_{n-1}}+\left[\partial_{u} \Phi_{\phi}, \Phi_{\theta_{n-1}}\right], \quad \partial_{w} \Phi_{\theta_{n}}=\partial_{v} \Phi_{\theta_{n-1}}+\left[\partial_{w} \Phi_{\phi}, \Phi_{\theta_{n-1}}\right] .
$$

We can also write

$$
\Phi_{\partial_{u} \theta_{n}}=\Phi_{\partial_{\bar{w}} \theta_{n-1}}+\Phi_{\left\{\partial_{u} \phi, \Phi_{\theta_{n-1}}\right\}}, \quad \Phi_{\partial_{w} \theta_{n}}=\Phi_{\partial_{v} \theta_{n-1}}+\Phi_{\left\{\partial_{w} \phi, \Phi_{\theta_{n-1}}\right\}} .
$$

In analogy with the self-dual Yang-Mills case, we can take $\Phi_{\theta_{0}}$ as a constant Hamiltonian vector field, e.g., $\Phi_{\theta_{0}}=\partial_{u}-\partial_{w}$, so that $\Phi_{\theta_{1}}=\left[\Phi_{\phi}, \Phi_{\theta_{0}}\right]=\Phi_{\left\{\phi, \theta_{0}\right\}}$, etc. For these two examples, we have $\theta_{0}=u+w$ and $\theta_{1}=\phi_{w}-\phi_{u}$, respectively. Then the conserved currents are obtained from

$$
J(x, \lambda)=\Phi_{\theta}, \quad \text { such that }\left[\partial_{\mu}, J^{\mu}\right]=0 .
$$

The analogous statement to $(2.45)$ is

$$
\partial^{2} \theta_{n}+\left\{\partial_{w} \phi, \partial_{u} \theta_{n}\right\}+\left\{\partial_{w} \theta_{n}, \partial_{u} \phi\right\}=\left\{\partial^{2} \phi+\left\{\partial_{w} \phi, \partial_{u} \phi\right\}, \theta_{n-1}\right\}=0,
$$

or in terms of Hamiltonian vector fields

$$
\Phi_{\partial^{2} \theta_{n}+\left\{\partial_{w} \phi, \partial_{u} \theta_{n}\right\}+i\left\{\partial_{w} \theta_{n}, \partial_{u} \phi\right\}}=\left[\Phi_{\partial^{2} \phi+\left\{\partial_{w} \phi, \partial_{u} \phi\right\}}, \Phi_{\theta_{n-1}}\right]=0 .
$$

\section{Moyal deformations in gauge theory and gravity}

In the previous section, we reviewed the known double copy at the level of equations of motion in self-dual Yang-Mills theory and gravity, and also drew attention to integrability aspects that have not been previously considered in a double copy context. Our overall 
aim is to generalise this construction, and thus we shall proceed by studying the kinds of generalisation of the above theories that are possible. We will first look at Moyal deformations of our usual self-dual theories, a concept which first arose in alternative formulations of quantum mechanics $[103,104]$ as well as in the study of field theories in non-commutative spacetimes $[105,106]$. The main idea is to replace the standard product on functions with the star product $\star$, defined in our present case as follows:

$$
f \star g \equiv f \exp \left(\frac{i \hbar}{2} \stackrel{\leftrightarrow}{P}\right) g
$$

where we have defined the operator:

$$
\stackrel{\leftrightarrow}{P} \equiv \overleftarrow{\partial}_{w} \vec{\partial}_{u}-\overleftarrow{\partial}_{u} \vec{\partial}_{w}
$$

that appears in the Poisson bracket of eq. (2.20):

$$
\{f, g\} \equiv f \stackrel{\leftrightarrow}{P} g
$$

The star product is associative and non-commutative, and contains a deformation parameter $\hbar$, whose notation stems from the original context in (non-commutative) quantum theories, and whose interpretation will be clarified below. Its use in defining deformed equations of motion usually proceeds by defining the so-called Moyal bracket ${ }^{2}$

$$
\{f, g\}^{M} \equiv \frac{1}{i \hbar}(f \star g-g \star f),
$$

such that in the limit $\hbar \rightarrow 0$ we have

$$
\lim _{\hbar \rightarrow 0} f \star g=f g, \quad \lim _{\hbar \rightarrow 0}\{f, g\}^{M}=\{f, g\} .
$$

That is, the star product of two functions reduces to the conventional (commutative) product of functions, and the Moyal bracket to the Poisson bracket. By substituting eq. (3.1) into eq. (3.4) and Taylor expanding in $\hbar$, we may write a general expression for the Moyal bracket in terms of the Poisson bracket, namely

$$
\{f, g\}^{M}=\sum_{s=0}^{\infty} \frac{(-1)^{s} \hbar^{2 s}}{2^{2 s}(2 s+1) !} \sum_{j=0}^{2 s}(-1)^{j}\left(\begin{array}{c}
2 s \\
j
\end{array}\right)\left\{\partial_{w}^{2 s-j} \partial_{u}^{j} f, \partial_{w}^{j} \partial_{u}^{2 s-j} g\right\},
$$

or equivalently

$$
\{f, g\}^{M}=\sum_{s=0}^{\infty} \frac{(-1)^{s} \hbar^{2 s}}{2^{2 s}(2 s+1) !} \sum_{j=0}^{2 s+1}(-1)^{j}\left(\begin{array}{c}
2 s+1 \\
j
\end{array}\right)\left(\partial_{w}^{j} \partial_{u}^{2 s+1-j} f\right)\left(\partial_{w}^{2 s+1-j} \partial_{u}^{j} g\right) .
$$

In a similar fashion to the previous section, we can explore the algebraic properties of this object. First, one can show that for any three functions, the Moyal bracket satisfies the Jacobi identity

$$
\left\{\{f, g\}^{M}, h\right\}^{M}+\left\{\{g, h\}^{M}, f\right\}^{M}+\left\{\{h, g\}^{M}, f\right\}^{M}=0 .
$$

\footnotetext{
${ }^{2}$ Notice that this product is real (see, for instance, eq. (3.6)), even though the individual star products are not. This means that phases which may appear in some non-commutative theories do not occur here.
} 
Then, we consider the particular relation

$$
\left\{e^{-i p_{1} x}, e^{-i p_{2} x}\right\}^{M}=-X^{M}\left(p_{1}, p_{2}\right) e^{-i\left(p_{1}+p_{2}\right) x},
$$

where the structure constant $X^{M}\left(p_{1}, p_{2}\right)$ is given by

$$
X^{M}\left(p_{1}, p_{2}\right) \equiv X\left(p_{1}, p_{2}\right) \sum_{s=0}^{\infty} \frac{(-1)^{s} \hbar^{2 s}}{2^{2 s}(2 s+1) !} \sum_{j=0}^{2 s}(-1)^{j}\left(\begin{array}{c}
2 s \\
j
\end{array}\right)\left(p_{1 w} p_{2 u}\right)^{2 s-j}\left(p_{1 u} p_{2 w}\right)^{j}
$$

This is a generalisation of the quantity $X\left(p_{1}, p_{2}\right)$ encountered in eq. (2.14), and reduces smoothly to the latter in the appropriate limit:

$$
\lim _{\hbar \rightarrow 0} X^{M}\left(p_{1}, p_{2}\right)=X\left(p_{1}, p_{2}\right)
$$

Furthermore, using eqs. (3.8) and (3.10), it is easy to see that the structure constant $X^{M}$ also satisfies the Jacobi identity

$$
X^{M}\left(p_{1}, p_{2}\right) X^{M}\left(p_{1}+p_{2}, p_{3}\right)+X^{M}\left(p_{2}, p_{3}\right) X^{M}\left(p_{2}+p_{3}, p_{1}\right)+X^{M}\left(p_{3}, p_{1}\right) X^{M}\left(p_{3}+p_{1}, p_{2}\right)=0
$$

We can therefore use the deformed structure constant $X^{M}\left(p_{1}, p_{2}\right)$ as a building block in constructing generalised field theories that obey double copy relations, and will see a number of examples below.

Another useful realisation is the Moyal deformation of the Lie bracket. Naively, we could define this as

$$
[V, W]^{M} \equiv \sum_{s=0}^{\infty} \frac{(-1)^{s} \hbar^{2 s}}{2^{2 s}(2 s+1) !} \sum_{j=0}^{2 s}(-1)^{j}\left(\begin{array}{c}
2 s \\
j
\end{array}\right)\left[\partial_{w}^{2 s-j} \partial_{u}^{j} V, \partial_{w}^{j} \partial_{u}^{2 s-j} W\right]
$$

For vector fields $V$ and $W$, the derivatives inside the Lie bracket are understood as acting as $\partial_{u} V \equiv\left[\partial_{u}, V\right]$, etc. Generically, this idea is naive, because such a Moyal bracket does not in general satisfy the Jacobi identity. A particular case where it does indeed satisfy it is that of Hamiltonian vector fields,

$$
\begin{aligned}
{\left[\Phi_{f}, \Phi_{g}\right]^{M} } & =\sum_{s=0}^{\infty} \frac{(-1)^{s} \hbar^{2 s}}{2^{2 s}(2 s+1) !} \sum_{j=0}^{2 s}(-1)^{j}\left(\begin{array}{c}
2 s \\
j
\end{array}\right)\left[\partial_{w}^{2 s-j} \partial_{u}^{j} \Phi_{f}, \partial_{w}^{j} \partial_{u}^{2 s-j} \Phi_{g}\right] \\
& =\sum_{s=0}^{\infty} \frac{(-1)^{s} \hbar^{2 s}}{2^{2 s}(2 s+1) !} \sum_{j=0}^{2 s}(-1)^{j}\left(\begin{array}{c}
2 s \\
j
\end{array}\right) \Phi_{\left\{\partial_{w}^{2 s-j} \partial_{u}^{j} f, \partial_{w}^{j} \partial_{u}^{2 s-j} g\right\}} \\
& =\Phi_{\{f, g\}^{M}},
\end{aligned}
$$

such that, analogously to the Jacobi identity (3.8),

$$
\left[\left[\Phi_{f}, \Phi_{g}\right]^{M}, \Phi_{h}\right]^{M}+\left[\left[\Phi_{g}, \Phi_{h}\right]^{M}, \Phi_{f}\right]^{M}+\left[\left[\Phi_{h}, \Phi_{f}\right]^{M}, \Phi_{g}\right]^{M}=0
$$




\subsection{Deformed self-dual gravity}

As a first application of the above ideas, we will consider a Moyal deformation of the Plebański equation, which was investigated in $[108,119,120]$. The deformed Plebański (second heavenly) equation is

$$
\partial^{2} \phi+\left\{\partial_{w} \phi, \partial_{u} \phi\right\}^{M}=0
$$

In momentum space, this deformed equation can be expressed as

$$
\phi(k)=\frac{1}{2} \int đ p_{1} d p_{2} \frac{\delta\left(p_{1}+p_{2}-k\right)}{k^{2}} X^{M}\left(p_{1}, p_{2}\right) X\left(p_{1}, p_{2}\right) \phi\left(p_{1}\right) \phi\left(p_{2}\right),
$$

and it is clear from eq. (3.11) that the usual Plebański equation both in coordinate space (2.23) and in momentum space eq. (2.22) are recovered in the limit $\hbar \rightarrow 0$.

The form of eq. (3.17) is particularly appealing from a double copy point of view. That is, all of the theories considered in this paper have momentum-space integral equations whose kernels involve products of structure constants, and the present case can be obtained by a process analogous to the double copy of eq. (2.21), making instead the replacement

$$
f^{a b c} \rightarrow X^{M}\left(p_{1}, p_{2}\right),
$$

in eq. (2.15). Below, we will extend this philosophy to obtain a web of theories with certain desirable properties. Before doing so, however, it is interesting to examine the integrability of the theory of eq. (3.16). This has been investigated in [120, 121], where as usual one may address this question by formulating a Lax pair. There are two possible ways to do this, namely we may consider a Lax pair consisting of undeformed vectors satisfying a deformed bracket, or 'deformed vectors' satisfying an undeformed bracket. The two options are related to attributing $X\left(p_{1}, p_{2}\right)$ or $X^{M}\left(p_{1}, p_{2}\right)$ in eq. (3.17) to the Lax compatibility condition or to the Lax pair. For the undeformed Lax pair, we can take the results of eq. (2.33) and use (3.14) to show that they satisfy the deformed compatibility condition

$$
[L, M]^{M}=0
$$

if $\phi$ satisfies the deformed Plebański eq. (3.16).

The argument when using a deformed pair (but undeformed bracket) is more involved. First, let us explain the deformation of the vectors as follows. We can rewrite the Hamiltonian vector field $\Phi_{f}$ in the $(w, u)$ plane, given in eq. (2.35), using the Poisson operator (3.2) in the form

$$
\Phi_{f} \equiv f \stackrel{\leftrightarrow}{P}=f_{w} \partial_{u}-f_{u} \partial_{w}
$$

Clearly, this vector satisfies the relation

$$
\Phi_{f}(g)=f \stackrel{\leftrightarrow}{P} g=\{f, g\} .
$$

Now, following the Poisson bracket case, eq. (3.21), we can define (in a slight abuse of language) a deformed Hamiltonian vector field, $\Phi_{f}^{M}$, by

$$
\Phi_{f}^{M}(g) \equiv\{f, g\}^{M} .
$$


Then we have $[121,122]$

$$
\begin{aligned}
\Phi_{f}^{M} & =\frac{2}{\hbar} f \sin \left(\frac{\hbar}{2} \stackrel{\leftrightarrow}{P}\right) \\
& =\sum_{s=0}^{\infty} \frac{(-1)^{s} \hbar^{2 s}}{2^{2 s}(2 s+1) !} \sum_{j=0}^{2 s+1}(-1)^{j}\left(\begin{array}{c}
2 s+1 \\
j
\end{array}\right)\left(\partial_{w}^{j} \partial_{u}^{2 s+1-j} f\right) \partial_{w}^{2 s+1-j} \partial_{u}^{j}
\end{aligned}
$$

Note that in the $\hbar \rightarrow 0$ limit, we recover a conventional Hamiltonian vector field, i.e. $\lim _{\hbar \rightarrow 0} \Phi_{f}^{M}=\Phi_{f}$. It follows from the above that the Lie bracket of deformed Hamiltonian vector fields is

$$
\left[\Phi_{f}^{M}, \Phi_{g}^{M}\right]=\Phi_{\{f, g\}^{M}}^{M} .
$$

Note that, following the Jacobi identity (3.8), the deformed Hamiltonian vector fields satisfies the Jacobi identity

$$
\left[\left[\Phi_{f}^{M}, \Phi_{g}^{M}\right], \Phi_{h}^{M}\right]+\left[\left[\Phi_{g}^{M}, \Phi_{h}^{M}\right], \Phi_{f}^{M}\right]+\left[\left[\Phi_{h}^{M}, \Phi_{f}^{M}\right], \Phi_{g}^{M}\right]=0 .
$$

Taking the 'deformed vectors'

$$
L^{M}=\partial_{u}-\lambda\left(\partial_{\bar{w}}+\Phi_{\phi_{u}}^{M}\right), \quad \quad M^{M}=\partial_{w}-\lambda\left(\partial_{v}+\Phi_{\phi_{w}}^{M}\right),
$$

we can check that they satisfy the condition

$$
\left[L^{M}, M^{M}\right]=0
$$

if $\phi$ satisfies the deformed Plebański eq. (3.16). To see this, we note that only the $\lambda^{2}$ term does not vanish trivially, taking the form

$$
\begin{aligned}
{\left[\partial_{v}+\Phi_{\phi_{w}}^{M}, \partial_{\bar{w}}+\Phi_{\phi_{u}}^{M}\right] } & =\partial^{2} \Phi_{\phi}^{M}+\left[\Phi_{\phi_{w}}^{M}, \Phi_{\phi_{u}}^{M}\right] \\
& =\Phi_{\partial^{2} \phi+\left\{\phi_{w}, \phi_{u}\right\}^{M}}^{M} \\
& =0 .
\end{aligned}
$$

In this case we have the deformed vectors

$$
\begin{aligned}
U & =\partial_{u}, & V & =\partial_{v}+\Phi_{\phi_{w}}^{M}, \\
W & =\partial_{w}, & \bar{W} & =\partial_{\bar{w}}+\Phi_{\phi_{u}}^{M} .
\end{aligned}
$$

The two equivalent approaches mentioned above, of deforming either the bracket or the Lax pair, are represented by (3.19) and (3.27).

We now proceed in analogy to section 2.3 to obtain an infinite tower of conserved currents for the deformed theory. First, the linearised equation of motion is obtained from the deformed Plebański equation (3.16) via the replacement $\Phi_{\phi}^{M} \rightarrow \Phi_{\phi}^{M}+\Phi_{\theta}^{M}$, is

$$
\partial^{2} \Phi_{\theta}^{M}+\left[\partial_{w} \Phi_{\phi}^{M}, \partial_{u} \Phi_{\theta}^{M}\right]+\left[\partial_{w} \Phi_{\theta}^{M}, \partial_{u} \Phi_{\phi}^{M}\right]=0 .
$$

Now, let us consider $\Phi_{\theta}^{M}$ of the form

$$
\Phi_{\theta}^{M}=\sum_{n=0}^{\infty} \Phi_{\theta_{n}}^{M} \lambda^{n} .
$$


We can express the infinite tower of linearised solutions $\left\{\Phi_{\theta_{n}}^{M}\right\}$ to eq. (3.30) recursively via

$$
\partial_{u} \Phi_{\theta_{n}}^{M}=\partial_{\bar{w}} \Phi_{\theta_{n-1}}^{M}+\left[\partial_{u} \Phi_{\phi}^{M}, \Phi_{\theta_{n-1}}^{M}\right], \quad \partial_{w} \Phi_{\theta_{n}}^{M}=\partial_{v} \Phi_{\theta_{n-1}}^{M}+\left[\partial_{w} \Phi_{\phi}^{M}, \Phi_{\theta_{n-1}}^{M}\right],
$$

with $\Phi_{\theta_{-1}}^{M} \equiv 0$. This $\Phi_{\theta_{n}}^{M}$ satisfies the compatibility condition $\partial_{u} \partial_{w} \Phi_{\theta_{n}}^{M}=\partial_{w} \partial_{u} \Phi_{\theta_{n}}^{M}$ and it is a solution of the linearised equation (3.30), using the Jacobi identity (3.25). Alternatively, the same infinite tower of linearised solutions can be obtained from Hamiltonian vector fields but using the deformed bracket. In this notation, we have the recursive equations

$$
\partial_{u} \Phi_{\theta_{n}}=\partial_{\bar{w}} \Phi_{\theta_{n-1}}+\left[\partial_{u} \Phi_{\phi}, \Phi_{\theta_{n-1}}\right]^{M}, \quad \partial_{w} \Phi_{\theta_{n}}=\partial_{v} \Phi_{\theta_{n-1}}+\left[\partial_{w} \Phi_{\phi}, \Phi_{\theta_{n-1}}\right]^{M},
$$

with $\Phi_{\theta_{-1}} \equiv 0$. As in the deformed Hamiltonian vector fields, this $\Phi_{\theta_{n}}$ satisfies the compatibility condition $\partial_{u} \partial_{w} \Phi_{\theta_{n}}=\partial_{w} \partial_{u} \Phi_{\theta_{n}}$ and it is a solution of the linearised equation

$$
\partial^{2} \Phi_{\theta}+\left[\partial_{w} \Phi_{\phi}, \partial_{u} \Phi_{\theta}\right]^{M}+\left[\partial_{w} \Phi_{\theta}, \partial_{u} \Phi_{\phi}\right]^{M}=0
$$

using the Jacobi identity (3.15).

The Moyal-deformed self-dual gravity shares features and properties with the undeformed version. One such property, as discussed above, is integrability. In terms of the language of the first heavenly equation, eq. (2.26), this property can be expressed in a concise geometric way with an associated 2 -form $\boldsymbol{\Omega}$, which satisfies the equations $d \boldsymbol{\Omega}=0$ and $\boldsymbol{\Omega} \wedge \boldsymbol{\Omega}=0$ [121]. This is the Kähler form, which is related to the Kähler potential $\Omega$ as $\boldsymbol{\Omega}=\Omega_{\mu \nu} d x^{\mu} \wedge d \tilde{x}^{\nu}$, where $x^{\mu}=\{w, u\}$ and $\tilde{x}^{\nu}=\{y, z\}$. In [123], a Moyal deformation of the first heavenly equation was considered, and a four-dimensional Moyal deformed integrable Kähler manifold was constructed by imposing in a consistent way that the deformed 2-form $\boldsymbol{\Omega}$ is closed, Hermitian and has unit determinant. This procedure provides an alternative but equivalent approach to equation (3.16), based on the second heavenly equation.

\subsubsection{Deformed self-dual gravity as undeformed self-dual Yang-Mills}

In this subsection, we take an alternative approach to the Moyal deformation of self-dual gravity, which leads directly to undeformed self-dual Yang-Mills. Instead of the deformation $X\left(p_{1}, p_{2}\right) \rightarrow X^{M}\left(p_{1}, p_{2}\right)$ considered above, we will see that we can think of $X\left(p_{1}, p_{2}\right) \rightarrow f^{a b c}$ as a Moyal deformation, at least in a particular construction.

In order to do that we follow now ref. [124], which considered the case of SDYM in $\mathbb{R}^{1, D-1}$ with $\operatorname{su}(N)$ or $\operatorname{sl}(N, \mathbb{C})$ Lie algebra valued fields. ${ }^{3}$ In ref. [124], a novel version of the basis for these algebras was found, in terms of a double index notation $m=\left(m_{1}, m_{2}\right)$. In more detail, the Lie algebra generators can be written as

$$
L_{m} \equiv-\frac{N}{2 \pi} \omega^{\frac{m_{1} m_{2}}{2}} S^{m_{1}} T^{m_{2}},
$$

where $\omega=\exp \left(\frac{2 \pi i}{N}\right)$, and $S$ and $T$ are $N \times N$ matrices such that $S^{N}=T^{N}=-1$ and $T \cdot S=\omega S \cdot T$. These generators satisfy

$$
\left[L_{m}, L_{n}\right]=i \mathcal{F}_{m, n}^{(m+n)} L_{m+n},
$$

\footnotetext{
${ }^{3}$ By SDYM beyond $D=4$, we mean simply the straightforward extension of $(2.11)$, where the wave operator is $D$-dimensional. The dimensionality is not relevant for our purposes.
} 
where $\mathcal{F}_{m, n}^{(m+n)}=\frac{N}{\pi} \sin \left(\frac{\pi}{N} m \times n\right)$. Thus, the analogue of eq. (2.15) is given by

$$
\Phi^{m+n}(k)=\frac{1}{2} \int đ p_{1} đ p_{2} \frac{\delta\left(p_{1}+p_{2}-k\right)}{k^{2}} X\left(p_{1}, p_{2}\right) \frac{N}{\pi} \sin \left(\frac{\pi}{N} m \times n\right) \Phi^{m}\left(p_{1}\right) \Phi^{n}\left(p_{2}\right) .
$$

In this basis, scalars $\Phi$ are expanded as $\Phi(x)=\sum_{m} \Phi^{m}(x) L_{m}$.

In the limit $N \rightarrow \infty$ for the Lie algebra in eq. (3.36), we get the $\operatorname{sdiff}\left(T^{2}\right)$ algebra

$$
\left\{e_{m}, e_{n}\right\}_{T}=(m \times n) e_{m+n},
$$

where $m \times n=m_{1} n_{2}-m_{2} n_{1}$, and the Poisson bracket on $T^{2}$ is $\{f, g\}_{T} \equiv f_{x_{2}} g_{x_{1}}-f_{x_{1}} g_{x_{2}}$. The generators of $\operatorname{sdiff}\left(T^{2}\right)$ with local coordinates $\left(x_{1}, x_{2}\right)$ are

$$
e_{m} \equiv \exp i\left(m_{1} x_{1}+m_{2} x_{2}\right)
$$

Furthermore there is an isomorphism which maps the Lie bracket (3.36) into the Moyal bracket for $e_{m}$ :

$$
\left\{e_{m}, e_{n}\right\}_{T}^{M}=\frac{2}{\hbar} \sin \left(\frac{\hbar}{2} m \times n\right) e_{m+n},
$$

where we have made the identification

$$
\hbar=\frac{2 \pi}{N}
$$

Thus the large- $N$ limit corresponds to the limit $\hbar \rightarrow 0$.

Then, the equation of motion on $\mathbb{R}^{1, D-3} \times T^{2}$ describes an integrable Moyal deformation of self-dual gravity equation known as the $\star$-SDYM system $[125,126]$ given by

$$
\partial^{2} \phi+\left\{\partial_{w} \phi, \partial_{u} \phi\right\}_{T}^{M}=0
$$

for $\phi=\sum_{m} \phi^{m}\left(\hbar ; \boldsymbol{x}, x_{1}, x_{2}\right) e_{m}$ and $\phi^{m}=\sum_{n=0}^{\infty} \hbar^{n} \phi_{n}^{m}$. Equation (3.42) can then be written as

$$
\phi^{m+n}(k)=\frac{1}{\hbar} \int đ p_{1} đ p_{2} \frac{\delta\left(p_{1}+p_{2}-k\right)}{k^{2}} X\left(p_{1}, p_{2}\right) \sin \left(\frac{\hbar}{2} m \times n\right) \phi^{m}\left(p_{1}\right) \phi^{n}\left(p_{2}\right) .
$$

The master equation (3.42) is proven to correspond to an integrable system [125], by showing the existence of Lax pairs, an infinite hierarchy of conserved quantities, and a twistor construction. Moreover in ref. [126] some explicit examples are given. The direct map between (3.43) and the SDYM equation (3.37) is clear.

Taking the limit $\hbar \rightarrow 0$ in eq. (3.43), we get the Plebański-Przanowski equation [119]

$$
\phi_{0}^{m+n}(k)=\frac{1}{2} \int đ p_{1} đ p_{2} \frac{\delta\left(p_{1}+p_{2}-k\right)}{k^{2}} X\left(p_{1}, p_{2}\right)(m \times n) \phi_{0}^{m}\left(p_{1}\right) \phi_{0}^{n}\left(p_{2}\right) .
$$

Equivalently, one can take the limit $N \rightarrow \infty$ in eq. (3.37) on $\mathbb{R}^{1, D-3}$. For instance, the $D=4$ case corresponds to $\mathbb{R}^{1,1} \times T^{2}$. In this case the gauge theory is the principal chiral model, which is an integrable two-dimensional reduction of SDYM equations on $\mathbb{R}^{1,3}$. This model leads to the Husain-Park heavenly equation which was discussed in [126, 127]. 
It is worth mentioning that this procedure can be employed to interpolate between all theories shown in figure 1. For instance, we can start from the biadjoint scalar theory and take in eq. (2.25) two times the representation of the Lie algebra in the trigonometric basis [124] i.e. $f^{a b c} \rightarrow \frac{2}{\hbar} \sin \left(\frac{\hbar}{2} m \times n\right)$ and $\widetilde{f}^{a^{\prime} b^{\prime} c^{\prime}} \rightarrow \frac{2}{\hbar^{\prime}} \sin \left(\frac{\hbar^{\prime}}{2} m^{\prime} \times n^{\prime}\right)$. Taking the limit, for instance, $\hbar \rightarrow 0$ this represents the inverse zeroth copy and we get self-dual Yang-Mills equation (2.15). A further limit $\hbar^{\prime} \rightarrow 0$ leads straightforwardly to the double copy, i.e. the self-dual gravity equation (2.22). It is also possible to start from the self-dual gravity equation (2.22) and take its single copy as a Moyal deformation of gravity with $\hbar^{\prime} \neq 0$. A further Moyal deformation with $\hbar \neq 0$ in the gauge theory leads directly to the zeroth copy giving the biadjoint scalar theory. Thus, this procedure allows us to interpolate with two continuous parameters $\hbar$ and $\hbar^{\prime}$ among the set of theories mentioned in figure 1. Of course, this particular construction relied on periodic identifications of coordinates, so that a $T^{2}$ arises, and the Fourier modes are discrete. If they are not discrete, then we will have structure constants labeled by continuous indices like $X^{M}\left(p_{1}, p_{2}\right)$ in the earlier discussion.

\subsection{Deformed self-dual Yang-Mills}

We will now explore the Moyal deformation of self-dual Yang-Mills theory. We will see, however, that the deformation that fits in with the colour-kinematics duality does not coincide with the most commonly considered Moyal deformation of self-dual Yang-Mills whereas the latter deformation is integrable [128, 129], the former does not preserve it.

Starting from the deformed Plebański equation for self-dual gravity in momentum space (3.17), we have two different single copies. One of them, replacing $X^{M}\left(p_{1}, p_{2}\right) \rightarrow f^{a b c}$, is the usual self-dual Yang-Mills theory. On the other hand, we can take $X\left(p_{1}, p_{2}\right) \rightarrow f^{a b c}$ and we obtain a deformed version of self-dual Yang-Mills. The equation of motion for this gauge theory in momentum space is

$$
\Phi^{a}(k)=\frac{1}{2} \int đ p_{1} đ p_{2} \frac{\delta\left(p_{1}+p_{2}-k\right)}{k^{2}} X^{M}\left(p_{1}, p_{2}\right) f^{a b c} \Phi^{b}\left(p_{1}\right) \Phi^{c}\left(p_{2}\right) .
$$

In the limit $\hbar \rightarrow 0$, we recover the usual SDYM equation (2.15). Furthermore, one may show that eq. (3.45) is equivalent to the position space equation

$$
\partial^{2} \Phi+i\left[\partial_{w} \Phi, \partial_{u} \Phi\right]^{M}=0
$$

where we are using the Moyal bracket (3.13). This theory differs from the usual Moyal deformation of self-dual Yang-Mills theory that is considered in the literature, which can be written as

$$
\partial^{2} \Phi+i\left[\partial_{w} \Phi, \partial_{u} \Phi\right]_{\star}=0, \quad[A, B]_{\star} \equiv A \star B-B \star A .
$$

This latter equation has a very specific interpretation: it is the theory one obtains upon introducing self-dual Yang-Mills theory in a non-commutative spacetime. To our knowledge, no such interpretation exists for eq. (3.46), which is the unique theory one obtains upon single copying the deformed Plebański equation in the above-mentioned fashion. Both eq. (3.46) and eq. (3.47) reduce to the usual self-dual Yang-Mills theory in the limit $\hbar \rightarrow 0$. The relation between these two distinct deformations is not obvious. In fact, the colour 
structure of eq. (3.47) is very different from the one typically found in gauge theories. For instance, a calculation shows that

$$
\begin{aligned}
i\left[\partial_{w}\left(\Phi^{a} T^{a}\right), \partial_{u}\left(\Phi^{b} T^{b}\right)\right]_{\star} & =-\frac{1}{2} f^{a b c} T^{c} \frac{d}{d \hbar}\left(\hbar\left\{\Phi^{a}, \Phi^{b}\right\}^{M}\right)-\hbar T^{(a} T^{b)}\left\{\partial_{w} \Phi^{a}, \partial_{u} \Phi^{b}\right\}^{M} \\
& =-\frac{1}{2} f^{a b c} T^{c}\left\{\Phi^{a}, \Phi^{b}\right\}-\hbar T^{(a} T^{b)}\left\{\partial_{w} \Phi^{a}, \partial_{u} \Phi^{b}\right\}+\mathcal{O}\left(\hbar^{2}\right) .
\end{aligned}
$$

In the right-hand side of the first line, the first/second term has only odd/even powers of $\hbar$. We can see right away that $\Phi$ is generically not a colour Lie algebra element in the usual Moyal deformation (3.47), i.e., $\Phi \neq \Phi^{a} T^{a}$; because if you start with $\Phi=\Phi^{a} T^{a}$ in the left-hand side of eq. (3.47), a distinct colour structure is generated, $T^{(a} T^{b)}$, which is not an element of the Lie algebra. Instead, $\Phi$ lives in the enveloping algebra generated by elements $T^{\left(a_{1}\right.} T^{a_{2}} \cdots T^{\left.a_{n}\right)}$. This contrasts with the single copy theory case of eq. (3.46), where $\Phi$ lives in the Lie algebra. The question of whether there is a more direct physical interpretation of eq. (3.46) is interesting. ${ }^{4}$ For our present purposes, we shall simply examine the question of integrability, by attempting to construct a Lax pair following an analogous process to that of SDYM. We will take a naive approach in order to see where it fails. Applying the Moyal bracket (3.13) for a gauge field, one may first look at the components of the deformed field strength tensor

$$
F_{\mu \nu}^{M} \equiv \partial_{\mu} A_{\nu}-\partial_{\nu} A_{\mu}-i\left[A_{\mu}, A_{\nu}\right]^{M},
$$

and the deformed self-duality conditions take the form

$$
\begin{aligned}
F_{u w}^{M} & =0, \\
F_{v \bar{w}}^{M} & =0, \\
F_{u v}^{M}-F_{w \bar{w}}^{M} & =0 .
\end{aligned}
$$

These equations arise as the condition $[L, M]^{M}=0$ for the two operators defined in eq. (2.30). However, this does not by itself guarantee integrability, which may be seen upon trying to construct a hierarchy of linearised solutions. The replacement $\Phi \rightarrow \Phi+\Psi$ results in the linearised equation

$$
\partial^{2} \Psi+i\left[\partial_{w} \Psi, \partial_{u} \Phi\right]^{M}+i\left[\partial_{w} \Phi, \partial_{u} \Psi\right]^{M}=0 .
$$

Expanding $\Psi$ in powers of the spectral parameter $\lambda$, we can express the infinite tower $\left\{\Psi_{n}\right\}$ recursively via

$$
\partial_{u} \Psi_{n}=\partial_{\bar{w}} \Psi_{n-1}+i\left[\partial_{u} \Phi, \Psi_{n-1}\right]^{M}, \quad \partial_{w} \Psi_{n}=\partial_{v} \Psi_{n-1}+i\left[\partial_{w} \Phi, \Psi_{n-1}\right]^{M},
$$

with $\Psi_{-1} \equiv 0$. One may be tempted to conclude that the theory is integrable. However, this type of construction relies crucially on the Jacobi identity for the bracket, which fails in the present case of the Moyal bracket (3.13). ${ }^{5}$ It relies on the Jacobi identity in order to

\footnotetext{
${ }^{4}$ There are several instances in the double copy literature where one theory of no obvious relevance of its own is very useful for providing building blocks, via the double copy, for more physically relevant theories.

${ }^{5}$ We made comments regarding this property near (3.13), where we noted that, when the Moyal-deformed bracket was applied to vector fields, the Jacobi identity did not apply in general, but did apply to Hamiltonian vector fields.
} 
prove that this infinite tower is a solution of the linearised equation (3.51) - without the Jacobi identity, one cannot show that $\Psi_{n}$ is a symmetry if $\Psi_{n-1}$ is. Even before that, the Jacobi identity is crucial in order to relate the linearised equation to the presumptive Lax pair. To see in detail how the Jacobi identity fails, let us take three gauge fields $V=V^{a} T^{a}$, $W=W^{b} T^{b}$ and $U=U^{c} T^{c}$. First, the Moyal bracket of $V$ and $W$ is explicitly

$$
\begin{aligned}
{[V, W]^{M} } & =i \sum_{s=0}^{\infty} \frac{(-1)^{s} \hbar^{2 s}}{2^{2 s}(2 s+1) !} \sum_{j=0}^{2 s}(-1)^{j}\left(\begin{array}{c}
2 s \\
j
\end{array}\right) f^{a b d}\left(\partial_{w}^{2 s-j} \partial_{u}^{j} V^{a}\right)\left(\partial_{w}^{j} \partial_{u}^{2 s-j} W^{b}\right) T^{d} \\
& =i \sum_{s=0}^{\infty} \frac{(-1)^{s} \hbar^{2 s}}{2^{2 s}(2 s+1) !} \sum_{j=0}^{2 s}(-1)^{j}\left(\begin{array}{c}
2 s \\
j
\end{array}\right) f^{a b d} V_{2 s-j, j}^{a} W_{j, 2 s-j}^{b} T^{d} \\
& =i f^{a b d} V^{a} W^{b} T^{d}+\mathcal{O}\left(\hbar^{2}\right)
\end{aligned}
$$

using the notation $\partial_{w}^{n} \partial_{u}^{m} F=F_{n, m}$. Now, for three gauge fields, we have

$$
\begin{aligned}
& {\left[[V, W]^{M}, U\right]^{M}=-f^{a b d} f^{d c e} T^{e}} \\
& \times \sum_{s, s^{\prime}=0}^{\infty} \frac{(-1)^{s+s^{\prime}} \hbar^{2\left(s+s^{\prime}\right)}}{2^{2\left(s+s^{\prime}\right)}(2 s+1) !\left(2 s^{\prime}+1\right) !} \sum_{j=0}^{2 s} \sum_{j^{\prime}=0}^{2 s^{\prime}}(-1)^{j+j^{\prime}}\left(\begin{array}{c}
2 s \\
j
\end{array}\right)\left(\begin{array}{c}
2 s^{\prime} \\
j^{\prime}
\end{array}\right)\left(V_{2 s-j, j}^{a} W_{j, 2 s-j}^{b}\right)_{2 s^{\prime}-j^{\prime}, j^{\prime}} U_{j^{\prime}, 2 s^{\prime}-j^{\prime}}^{c} \\
& =-f^{a b d} f^{d c e} T^{e}\left[V^{a} W^{b} U^{c}-\frac{\hbar^{2}}{24} \sum_{j=0}^{2}(-1)^{j}\left(\begin{array}{c}
2 \\
j
\end{array}\right)\left(V_{2-j, j}^{a} W_{j, 2-j}^{b} U^{c}+\left(V^{a} W^{b}\right)_{2-j, j} U_{j, 2-j}^{c}\right)\right]+\mathcal{O}\left(\hbar^{4}\right) .
\end{aligned}
$$

Finally, the Jacobi type equation gives

$$
\begin{aligned}
{\left[[V, W]^{M}, U\right]^{M}+\left[[W, U]^{M}, V\right]^{M}+\left[[U, V]^{M}, W\right]^{M} } & \\
& =-\left(f^{a b d} f^{d c e}+f^{b c d} f^{d a e}+f^{c a d} f^{d b e}\right) T^{e} V^{a} W^{b} U^{c}+\mathcal{O}\left(\hbar^{2}\right) .
\end{aligned}
$$

The leading order term, $\mathcal{O}\left(\hbar^{0}\right)$, vanishes due to the colour Jacobi identity. However, the first subleading term is proportional to

$$
\begin{aligned}
& \sum_{j=0}^{2}(-1)^{j}\left(\begin{array}{l}
2 \\
j
\end{array}\right)\left[f^{a b d} f^{d c e}\left(V_{2-j, j}^{a} W_{j, 2-j}^{b} U^{c}+\left(V^{a} W^{b}\right)_{2-j, j} U_{j, 2-j}^{c}\right)\right. \\
& \left.+f^{b c d} f^{d a e}\left(W_{2-j, j}^{b} U_{j, 2-j}^{c} V^{a}+\left(W^{b} U^{c}\right)_{2-j, j} V_{j, 2-j}^{a}\right)+f^{c a d} f^{d b e}\left(U_{2-j, j}^{c} V_{j, 2-j}^{a} W^{b}+\left(U^{c} V^{a}\right)_{2-j, j} W_{j, 2-j}^{b}\right)\right] \\
& \neq 0
\end{aligned}
$$

that is, it does not vanish generically. Hence, $\left[[V, W]^{M}, U\right]^{M}+\left[[W, U]^{M}, V\right]^{M}+$ $\left[[U, V]^{M}, W\right]^{M} \neq 0$. The integrability property relies implicitly on the Jacobi identity for the appropriate bracket, which fails in this case. Therefore, the deformation (3.46) of the SDYM equation of motion, which we arrived at by taking the single copy of Moyaldeformed SDG that keeps the factor $X^{M}\left(p_{1}, p_{2}\right)$, and changes $X\left(p_{1}, p_{2}\right) \rightarrow f^{a b c}$, is not integrable. This is because the integrability of Moyal-deformed SDG relies precisely on the factor $X\left(p_{1}, p_{2}\right)$, which we discarded here.

\subsection{Doubly deformed self-dual gravity}

We have so far been constructing various theories from known self-dual integral equations, by replacing different structure constants by their deformed counterparts. Continuing 
this procedure, we can consider the theory one obtains by taking two copies of the Moyaldeformed kinematic algebra, whose structure constant is $X^{M}\left(p_{1}, p_{2}\right)$. This can be obtained e.g. by taking the deformed self-dual Yang-Mills theory of eq. (3.45), and replacing $f^{a b c} \rightarrow$ $X^{M^{\prime}}\left(p_{1}, p_{2}\right)$. The resulting equation of motion in momentum space then takes the form

$$
\phi(k)=\frac{1}{2} \int d p_{1} d p_{2} \frac{\delta\left(p_{1}+p_{2}-k\right)}{k^{2}} X^{M}\left(p_{1}, p_{2}\right) X^{M^{\prime}}\left(p_{1}, p_{2}\right) \phi\left(p_{1}\right) \phi\left(p_{2}\right),
$$

where

$$
X^{M^{\prime}}\left(p_{1}, p_{2}\right)=X\left(p_{1}, p_{2}\right) \sum_{s=0}^{\infty} \frac{(-1)^{s} \hbar^{2 s}}{2^{2 s}(2 s+1) !} \sum_{j=0}^{2 s}(-1)^{j}\left(\begin{array}{c}
2 s \\
j
\end{array}\right)\left(p_{1 w} p_{2 u}\right)^{2 s-j}\left(p_{1 u} p_{2 w}\right)^{j},
$$

and we have introduced a second deformation parameter $\hbar^{\prime}$ to be associated with $M^{\prime}$. In position space this equation is

$$
\partial^{2} \phi+\sum_{s=0}^{\infty} \frac{(-1)^{s} \hbar^{2 s}}{2^{2 s}(2 s+1) !} \sum_{j=0}^{2 s}(-1)^{j}\left(\begin{array}{c}
2 s \\
j
\end{array}\right)\left\{\partial_{w}^{2 s-j+1} \partial_{u}^{j} \phi, \partial_{w}^{j} \partial_{u}^{2 s-j+1} \phi\right\}^{M^{\prime}}=0 .
$$

If we take either of the limits $\hbar \rightarrow 0$ or $\hbar^{\prime} \rightarrow 0$, we recover the deformed Plebański equation (3.16). Furthermore, it is straightforward to verify that eq. (3.59) arises from the (doubly deformed) Lax Pair condition

$$
\left[L^{M^{\prime}}, M^{M^{\prime}}\right]^{M}=0 .
$$

To see this, one may first note that only the $\lambda^{2}$ does not vanish trivially, which in turn leads explicitly to

$$
\begin{aligned}
{\left[\partial_{v}+\Phi_{\phi_{w}}^{M^{\prime}}, \partial_{\bar{w}}+\Phi_{\phi_{u}}^{M^{\prime}}\right]^{M} } & =\partial^{2} \Phi_{\phi}^{M^{\prime}}+\left[\Phi_{\phi_{w}}^{M^{\prime}}, \Phi_{\phi_{u}}^{M^{\prime}}\right]^{M} \\
& =\Phi_{\partial^{2} \phi+\sum_{s=0}^{M^{\prime}} \frac{(-1)^{s} \hbar^{2 s}}{2^{2 s}(2 s+1) !} \sum_{j=0}^{2 s}(-1)^{j}\left(\begin{array}{c}
2 s \\
j
\end{array}\right)\left\{\partial_{w}^{2 s+1-j} \partial_{u}^{j} \phi, \partial_{w}^{j} \partial_{u}^{2 s+1-j} \phi\right\}^{M}} \\
& =0 .
\end{aligned}
$$

Moreover, we can obtain the same equation with $\left[L^{M}, M^{M}\right]^{M^{\prime}}=0$. For this double deformed Plebański equation (3.59), we obtain a linearised equation of motion obtained from the replacement $\Phi_{\phi}^{M^{\prime}} \rightarrow \Phi_{\phi}^{M^{\prime}}+\Phi_{\theta}^{M^{\prime}}$. Expanding $\Phi_{\theta}^{M^{\prime}}$ in powers of the spectral parameter $\lambda$, the infinite tower $\left\{\Phi_{\theta_{n}}^{M^{\prime}}\right\}$ can be expressed recursively as

$$
\partial_{u} \Phi_{\theta_{n}}^{M^{\prime}}=\partial_{\bar{w}} \Phi_{\theta_{n-1}}^{M^{\prime}}+\left[\partial_{u} \Phi_{\phi}^{M^{\prime}}, \Phi_{\theta_{n-1}}^{M^{\prime}}\right]^{M}, \quad \partial_{w} \Phi_{\theta_{n}}^{M^{\prime}}=\partial_{v} \Phi_{\theta_{n-1}}^{M^{\prime}}+\left[\partial_{w} \Phi_{\phi}^{M^{\prime}}, \Phi_{\theta_{n-1}}^{M^{\prime}}\right]^{M},
$$

with $\Phi_{\theta_{-1}}^{M^{\prime}}=0$. Similar to the deformed self-dual Yang-Mills infinity tower (3.52), the infinity tower (3.62) satisfies the compatibility condition $\partial_{w} \partial_{u} \Phi_{\theta_{n}}^{M^{\prime}}=\partial_{u} \partial_{w} \Phi_{\theta_{n}}^{M M^{\prime}}$. However, also similarly to the deformed self-dual Yang-Mills theory, the integrability construction fails because the Jacobi identity of the Moyal bracket applied to deformed vector fields fails.

In this section, we have demonstrated the existence of a family of generalised selfdual gauge and gravity theories obeying double copy relationships. The key ingredients were the expression of such theories as integral equations whose kernels manifestly contain 
products of structure constants, and the introduction of new such constants - e.g., those of Moyal-deformed diffeomorphism algebra, $X^{M}\left(p_{1}, p_{2}\right)$ - that could be used as appropriate building blocks. In all cases, we could show that the position space equations of motion resulted from a Lax pair type of condition, although this was not always sufficient to guarantee integrability. The Moyal deformation considered above is in fact only one possible generalisation of the heavenly double copy between self-dual theories: we examine another in the following section.

\section{Generalising to the diffeomorphism algebra}

We will now, operating with a similar motive as in the previous section, consider a second — independent - generalisation of our usual self-dual theories. That is, we will consider a less restrictive gauge group, by relaxing the requirement that the kinematic group (SDiff) preserves the volume form, in this case the $(u, w)$-area form. This gives instead the group of full two-dimensional diffeomorphisms (Diff) in the $(u, w)$ plane. As we will see, this Diff group is in turn related to hyper-Hermitian manifolds, thus going beyond the hyper-Kähler structures in conventional self-dual gravity, discussed here in section 2.2.

Let us take a vector field $\Psi_{f}$ in the $(w, u)$ plane:

$$
\Psi_{f} \equiv f^{A} \partial_{A}=f^{w} \partial_{w}+f^{u} \partial_{u}
$$

where $A \in\{w, u\}$ and $f^{A}=f^{A}(u, v, w, \bar{w})$ depend on all the coordinates. In this form, $\Psi_{f}$ is an element of diff, the Lie algebra of Diff. The vector $\Psi_{f}$ satisfies the Jacobi identity

$$
\left[\left[\Psi_{f}, \Psi_{g}\right], \Psi_{h}\right]+\left[\left[\Psi_{g}, \Psi_{h}\right], \Psi_{f}\right]+\left[\left[\Psi_{h}, \Psi_{f}\right], \Psi_{g}\right]=0
$$

for the functions $f^{A}, g^{A}, h^{A}$. To make the connection with the previous sections, we need the kinematic object associated with the Lie algebra diff. To this end, let us define a vector in the direction $A$ and momentum $p$ by $\Psi_{p, A} \equiv e^{-i p x} \partial_{A}$. The commutator between two of such vectors is

$$
\left[\Psi_{p, A}, \Psi_{q, B}\right]=i Y^{C}\left(q_{A}, p_{B}\right) \Psi_{p+q, C}
$$

where we define the kinematic object

$$
Y^{A}\left(p_{1 C}, p_{2 B}\right) \equiv p_{2 B} \delta_{C}^{A}-p_{1 C} \delta_{B}^{A}
$$

This satisfies $Y^{A}\left(p_{1 C}, p_{2 B}\right)=-Y^{A}\left(p_{2 B}, p_{1 C}\right)$ and also, as we will see shortly, a Jacobi identity. As a consequence, it will be interpreted as a structure constant of the twodimensional diffeomorphism algebra (diff). Following eq. (4.2), we have the Jacobi relation

$$
Y^{D}\left(q_{A}, p_{B}\right) Y^{E}\left(k_{D},(p+q)_{C}\right)+Y^{D}\left(k_{B}, q_{C}\right) Y^{E}\left(p_{D},(q+k)_{A}\right)+Y^{D}\left(p_{C}, k_{A}\right) Y^{E}\left(q_{D},(p+k)_{B}\right)=0,
$$

for any $E$. The kinematic factor $Y^{A}\left(p_{1 C}, p_{2 B}\right)$, which is the structure constant for diff, will be our focus in this section. 


\subsection{Hyper-Hermitian manifold}

The notion of a hyper-Hermitian manifold is a generalisation of the hyper-Kähler case, where the volume preserving condition $\mathcal{L}_{L} v=\mathcal{L}_{M} v=0$ is relaxed on the gravitational Lax pair (2.31). Taking the Lax pair (2.31), we can rewrite the condition $[L, M]=0$ as

$$
[U, W]=0, \quad[U, V]+[\bar{W}, W]=0, \quad[\bar{W}, V]=0 .
$$

Then, $\mathbf{V}=\{U, V, W, \bar{W}\}$ forms a null tetrad for a hyper-Hermitian metric on a fourdimensional complex manifold $\mathcal{M}$. Following [116, 130, 131], we can define the vectors

$$
\begin{array}{ll}
U=\partial_{u}, & W=\partial_{w}, \\
V=\partial_{v}+\partial_{w} \Psi_{f}, & \bar{W}=\partial_{\bar{w}}+\partial_{u} \Psi_{f} .
\end{array}
$$

As in previous sections, for notational brevity, we denote $\partial_{w} \Psi_{f} \equiv\left[\partial_{w}, \Psi_{f}\right]$ and $\partial_{u} \Psi_{f} \equiv$ $\left[\partial_{u}, \Psi_{f}\right]$. The tetrad $\mathbf{V}$ satisfies eq. (4.6) if and only if $f^{A}$ satisfies

$$
\partial^{2} f^{A}+\left\{f^{B}, \partial_{B} f^{A}\right\}=0 .
$$

It was shown in ref. [116] that this system describes an hyper-Hermitian manifold. In momentum space, eq. (4.8) implies the integral equation

$$
f^{A}(k)=\frac{1}{2} \int đ p_{1} \varpi p_{2} \frac{\delta\left(p_{1}+p_{2}-k\right)}{k^{2}} X\left(p_{1}, p_{2}\right) Y^{A}\left(p_{1 C}, p_{2 B}\right) f^{B}\left(p_{1}\right) f^{C}\left(p_{2}\right),
$$

thus justifying our above remark relating the Diff group to hyper-Hermitian manifolds.

Before proceeding, let us point out that Hyper-Hermitian geometries can be more formally defined as follows. Let $\mathcal{M}$ be a four-dimensional manifold and $g$ be a Riemannian metric on $\mathcal{M}$. If $\mathcal{M}$ is equipped with three complex structures $I, J, K$ satisfying the algebra of quaternions, i.e., $I J=-J I=K$, and $g$ is a Hermitian metric for the three complex structures, then $\mathcal{M}$ is hyper-Hermitian. If, besides this, the three Kähler forms are closed, $d \Omega_{I}=d \Omega_{J}=d \Omega_{K}=0$, then $\mathcal{M}$ is hyper-Kähler. A hyper-Hermitian manifold has a self-dual Weyl tensor. On the other hand, a hyper-Kähler manifold has a self-dual Weyl tensor and also a vanishing Ricci tensor. ${ }^{6}$

Similarly to the arguments in section 3, the fact that the kernel of the integral equation of eq. (4.9) involves a product of structure constants immediately furnishes it with a double copy interpretation. In particular, we note that eq. (4.9) can be obtained via the doublecopy-like replacement

$$
f^{a b c} \rightarrow Y^{A}\left(p_{1 C}, p_{2 B}\right),
$$

in the structure constants of the colour Lie algebra in eq. (2.15). Furthermore, eq. (4.9) then shows that hyper-Hermitian manifolds are governed by a product of kinematic Lie algebras, namely

$$
\text { sdiff } \times \text { diff. }
$$

\footnotetext{
${ }^{6}$ These statements apply to our convention on (anti-)self-duality. Literature following different conventions may take the Weyl tensor for these manifolds to be anti-self-dual instead.
} 
Now, the Lax pair $L$ and $M$ associated with the vectors (4.7) for an hyper-Hermitian manifold is given by

$$
L=\partial_{u}-\lambda\left(\partial_{\bar{w}}+\partial_{u} \Psi_{f}\right), \quad M=\partial_{w}-\lambda\left(\partial_{v}+\partial_{w} \Psi_{f}\right),
$$

and the eq. (4.8) arises from the condition $[L, M]=0$. We can recover conventional selfdual gravity if we impose the condition $\mathcal{L}_{L} v=\mathcal{L}_{M} v=0$ on the Lax pair (4.11): there must then exist a function $\phi$ such that $f^{w}=-\partial_{u} \phi$ and $f^{u}=\partial_{w} \phi$, such that eq. (4.8) reduces to the single Plebański equation (2.23).

Similarly to the previous cases, we can construct a hierarchy of linearised solutions. The linearised hyper-Hermitian equation, for $\Psi_{f} \rightarrow \Psi_{f}+\Psi_{g}$, is

$$
\partial^{2} \Psi_{g}+\left[\partial_{w} \Psi_{f}, \partial_{u} \Psi_{g}\right]+\left[\partial_{w} \Psi_{g}, \partial_{u} \Psi_{f}\right]=0 .
$$

Expanding $\Psi_{g}$ in powers of $\lambda$, the infinite tower of linearised solutions $\left\{\Psi_{g_{n}}\right\}$ to (4.12) is recursively expressed as

$$
\partial_{u} \Psi_{g_{n}}=\partial_{\bar{w}} \Psi_{g_{n-1}}+\left[\partial_{u} \Psi_{f}, \Psi_{g_{n-1}}\right], \quad \partial_{w} \Psi_{g_{n}}=\partial_{v} \Psi_{g_{n-1}}+\left[\partial_{w} \Psi_{f}, \Psi_{g_{n-1}}\right],
$$

with $\Psi_{g_{-1}} \equiv 0$. This $\Psi_{g_{n}}$ satisfies the compatibility condition $\partial_{u} \partial_{w} \Psi_{g_{n}}=\partial_{w} \partial_{u} \Psi_{g_{n}}$, and the tower solves eq. (4.12). This relies on the Jacobi identity (4.2).

In addition to the structure constants of diff considered above, we could also introduce a Moyal deformation of sdiff. ${ }^{7}$ This results in a deformed version of the hyper-Hermitian equation (4.8). The integral form of the equation of motion reads

$$
f^{A}(k)=\frac{1}{2} \int d p_{1} d p_{2} \frac{\delta\left(p_{1}+p_{2}-k\right)}{k^{2}} X^{M}\left(p_{1}, p_{2}\right) Y^{A}\left(p_{1 C}, p_{2 B}\right) f^{B}\left(p_{1}\right) f^{C}\left(p_{2}\right),
$$

and it is straightforward to obtain the equation in position space,

$$
\partial^{2} f^{A}+\left\{f^{B}, \partial_{B} f^{A}\right\}^{M}=0 .
$$

Using the Lax pair eq. (4.11), this deformed equation arises from the condition $[L, M]^{M}=0$. The linearised deformed hyper-Hermitian equation, for $\Psi_{f} \rightarrow \Psi_{f}+\Psi_{g}$, is

$$
\partial^{2} \Psi_{g}+\left[\partial_{w} \Psi_{f}, \partial_{u} \Psi_{g}\right]^{M}+\left[\partial_{w} \Psi_{g}, \partial_{u} \Psi_{f}\right]^{M}=0 .
$$

Expanding $\Psi_{g}$ in power of $\lambda$ and using the Lax equation, the infinite tower $\left\{\Psi_{g_{n}}\right\}$ is recursively expressed as

$$
\partial_{u} \Psi_{g_{n}}=\partial_{\bar{w}} \Psi_{g_{n-1}}+\left[\partial_{u} \Psi_{f}, \Psi_{g_{n-1}}\right]^{M}, \quad \partial_{w} \Psi_{g_{n}}=\partial_{v} \Psi_{g_{n-1}}+\left[\partial_{w} \Psi_{f}, \Psi_{g_{n-1}}\right]^{M},
$$

with $\Psi_{g_{-1}} \equiv 0$. In this naive hierarchy we are using the Moyal bracket on non-Hamiltonian vector fields, and similarly to the deformed self-dual Yang-Mills and doubly deformed Plebański cases, the Jacobi identity fails, and hence integrability is lost. ${ }^{8}$

\footnotetext{
${ }^{7}$ Seen in a different way, we could start from eq. (3.45) and make the replacement (4.10).

${ }^{8} \mathrm{~A}$ different version of a deformed hyper-Hermitian structure is presented in [132]. That particular example formally retains integrability of the theory, although a twistor description is missing. Following [133], they make a deformation of the standard homomorphism between $\operatorname{diff}\left(S^{1}\right)$ and the Poisson algebra on the cotangent bundle $T^{*} S^{1}$. It would be interesting to know whether these ideas fit into an expanded double copy web of theories.
} 


\subsection{Other Diff theories}

Motivated by the appearance of the Diff group, a natural question is: what is the 'gauge theory' with kinematic algebra diff? This gauge theory is the single copy of the hyperHermitian eq. (4.9) (or its deformed version (4.14)), via the replacement $X\left(p_{1}, p_{2}\right) \rightarrow f^{a b c}$. The 'Diff-gauge' theory is described by the equation

$$
\Phi^{a A}(k)=\frac{1}{2} \int đ p_{1} đ p_{2} \frac{\delta\left(p_{1}+p_{2}-k\right)}{k^{2}} f^{a b c} Y^{A}\left(p_{1 C}, p_{2 B}\right) \Phi^{b B}\left(p_{1}\right) \Phi^{c C}\left(p_{2}\right),
$$

for $A=u, w$. It is straightforward to express it in position space as

$$
\partial^{2} \Phi^{A}+i\left[\Phi^{B}, \partial_{B} \Phi^{A}\right]=0 .
$$

We can recover the SDYM eq. (2.11) if we impose on $\Phi^{A}$ the condition $\Phi^{w}=-\partial_{u} \Phi$ and $\Phi^{u}=\partial_{w} \Phi$. Of course, this condition is similar to imposing the volume preserving condition in the hyper-Hermitian case.

Finally, we can make the replacement (4.10) in eq. (4.18) and obtain a theory with two copies of the diff algebra. In momentum space these equations are

$$
f^{A B}(k)=\frac{1}{2} \int đ p_{1} \Varangle p_{2} \frac{\delta\left(p_{1}+p_{2}-k\right)}{k^{2}} Y^{A}\left(p_{1 E}, p_{2 C}\right) Y^{B}\left(p_{1 F}, p_{2 D}\right) f^{C D}\left(p_{1}\right) f^{E F}\left(p_{2}\right),
$$

for $A, B, C, D=u, w$, whereas in position space, we have

$$
\partial^{2} f^{A B}+f^{C D}\left(\partial_{C} \partial_{D} f^{A B}\right)-\left(\partial_{C} f^{A D}\right)\left(\partial_{D} f^{C B}\right)=0 .
$$

Equations (4.18) and (4.20) complete our web of theories, by combining all of the modifications of self-dual Yang-Mills that we have considered. Again, the physical relevance of the new theories on their own - outside the remit of the double copy — is not clear, and it would be interesting to explore them further.

We pointed out early on the well-known fact that it is possible to recover self-dual gravity (hyper-Kähler manifold) as a symmetry reduction of the self-dual Yang-Mills theory, considering the gauge group as the area-preserving diffeomorphisms group on the twosurface $\Sigma, \operatorname{SDiff}(\Sigma)$ [108-110], and that this can be used to build hyper-Kähler metrics from solutions of two-dimensional reduced SDYM equations [111-113]. In a similar form, as seen in this section, we can obtain the hyper-Hermitian equations taking the gauge group as the area-preserving diffeomorphisms group in the Diff-gauge equations. It would be interesting to see if it is possible to build hyper-Hermitian metrics from solutions of two-dimensional reduced Diff-gauge equations.

\section{Summary of results}

Throughout this paper, we have established a set of theories that generalise self-dual YangMills and gravity. The momentum space equation for each theory contains a manifest product of structure constants associated with colour and / or kinematic algebras, thus allowing theories within the set to be related via the double copy. We summarise the complete set in table 1, where the first column (consisting of choosing two potentially distinct 


\begin{tabular}{|l|l|l|l|l|}
\hline$\times$ & $f^{a b c}(\mathrm{G})$ & $X\left(p_{1}, p_{2}\right)$ (sdiff) & $X^{M}\left(p_{1}, p_{2}\right)($ Moyal $)$ & $Y^{A}\left(p_{1 B}, p_{2 C}\right)($ diff $)$ \\
\hline$f^{a b c}(\mathrm{G})$ & $\begin{array}{l}\text { Biadjoint } \\
\text { scalar }\end{array}$ & SDYM & Moyal-deformed SDYM & Diff-Gauge \\
\hline $\begin{array}{l}X\left(p_{1}, p_{2}\right)(\mathrm{sd}- \\
\text { iff })\end{array}$ & - & $\begin{array}{l}\text { SDG Plebański } \\
(\text { Hyper-Kähler })\end{array}$ & $\begin{array}{l}\text { Moyal-deformed SDG } \\
(\text { Hyper-Kähler) }\end{array}$ & Hyper-Hermitian \\
\hline $\begin{array}{l}X^{M}\left(p_{1}, p_{2}\right) \\
(\text { Moyal })\end{array}$ & - & - & Moyal $\times$ Moyal SDG & $\begin{array}{l}\text { Moyal-deformed } \\
\text { Hyper-Hermitian }\end{array}$ \\
\hline $\begin{array}{l}Y^{A}\left(p_{1 B}, p_{2 C}\right) \\
(\text { diff })\end{array}$ & - & - & - & Diff $\times$ Diff Gravity \\
\hline
\end{tabular}

Table 1. The web of theories studied in this paper. Each theory has an integral equation containing two structure constants, appearing on the left-hand and upper entries of the table. We represented in grey the theories that are integrable.

colour algebras) corresponds to biadjoint scalar theory. The second column contains the usual SDYM and SDGR theories (for a hyper-Kähler manifold), whose double copy was discussed in detail in ref. [83]. The third column corresponds to our first generalisation of the double copy procedure (section 3), in which one of the structure constants is taken to be a Moyal deformation of the kinematic structure constant. This leads to single Moyal deformations of SDYM and SDGR, and also a doubly deformed gravity theory, whose equation of motion contains a product of two Moyal kinematic factors. We should point out the (non-integrable) Moyal-deformed SDYM case considered in the table, which is the one that straightforwardly matches the double copy structure, is not the same as the more conventional Moyal deformation of SDYM commonly considered in the literature, as discussed in section 3.2. The second generalisation considers the kinematic algebra diff, where the area preserving condition has been relaxed. A product with sdiff results in a Hyper-Hermitian manifold. In section 4, we have studied this as well as its Moyal deformation, a Diff-gauge theory, and a double-Diff gravity, as recapped in the fourth column of table 1.

The entries of table 1 identified in grey correspond to integrable theories. All such theories have $X\left(p_{1}, p_{2}\right)$ as one of the double copy factors, and this factor is at the origin of their integrability. The equation of motion in momentum space reads

$$
\Upsilon_{\rho}(p)=\frac{1}{2} \int đ p_{1} \succsim p_{2} \frac{\delta\left(p_{1}+p_{2}-p\right)}{p^{2}} X\left(p_{1}, p_{2}\right) \Pi_{12} \Upsilon_{\rho_{1}}\left(p_{1}\right) \Upsilon_{\rho_{2}}\left(p_{2}\right),
$$

where $\Pi_{12}$ provides the other structure constant. In coordinate space, we have

$$
\partial^{2} \Upsilon_{\rho}+\left[\partial_{w} \Upsilon_{\rho}, \partial_{u} \Upsilon_{\rho}\right]=0
$$

Pleasingly, all the integrable theories can be written in a similar manner, where $\Upsilon_{\rho}$ is $\Phi=\Phi^{a} T^{a}$ for self-dual Yang-Mills, is $\Phi_{\phi}$ for self-dual gravity, is $\Phi_{\phi}^{M}$ for singly Moyaldeformed self-dual gravity, and is $\Psi_{f}$ for hyper-Hermitian gravity. Their Lax pairs can be expressed as

$$
L=\partial_{u}-\lambda\left(\partial_{\bar{w}}+\partial_{u} \Upsilon_{\rho}\right), \quad M=\partial_{w}-\lambda\left(\partial_{v}+\partial_{w} \Upsilon_{\rho}\right),
$$

and the equation of motion follows from the condition $[L, M]=0$. Integrability follows from the existence of an infinite hierarchy of conserved currents, associated to an infinite 
hierarchy of solutions to the linearised equation of motion. The latter is obtained by making the replacement $\Upsilon_{\rho} \rightarrow \Upsilon_{\rho}+\Upsilon_{\alpha}$, in eq. (5.2):

$$
\partial^{2} \Upsilon_{\alpha}+\left[\partial_{w} \Upsilon_{\rho}, \partial_{u} \Upsilon_{\alpha}\right]+\left[\partial_{w} \Upsilon_{\alpha}, \partial_{u} \Upsilon_{\rho}\right]=0
$$

The hierarchy of solutions $\left\{\Upsilon_{\alpha_{n}}\right\}$ arises via

$$
\partial_{u} \Upsilon_{\alpha_{n}}=\partial_{\bar{w}} \Upsilon_{\alpha_{n-1}}+\left[\partial_{u} \Upsilon_{\rho}, \Upsilon_{\alpha_{n-1}}\right], \quad \partial_{w} \Upsilon_{\alpha_{n}}=\partial_{v} \Upsilon_{\alpha_{n-1}}+\left[\partial_{w} \Upsilon_{\rho}, \Upsilon_{\alpha_{n-1}}\right],
$$

where $\Upsilon_{\alpha_{-1}} \equiv 0$. In this hierarchy, $\Upsilon_{\alpha_{n}}$ is guaranteed to satisfy the linearised equation of motion if $\Upsilon_{\alpha_{n-1}}$ does.

Several non-integrable theories in table 1 naively share some of these features, at least those which possess $X^{M}\left(p_{1}, p_{2}\right)$ as one of the double copy factors (we exclude here the case where $X\left(p_{1}, p_{2}\right)$ is the other factor, where the theory is integrable). Then eq. (5.2) changes only by employing a Moyal-deformed bracket, that is, we make the substitution $[\cdot, \cdot] \rightarrow[\cdot, \cdot]^{M}$. The following equations also appear to tell an analogous story with the same substitution. However, crucially, the Moyal-deformed bracket fails to obey the Jacobi identity, and the steps leading to the conclusion of integrability fail.

\section{Conclusions}

In this paper, we have constructed new examples of gauge and gravity theories satisfying manifest double copy relations, motivated by previous work in the self-dual sector of Yang-Mills theory and gravity [83]. Each of our theories has an integral momentum-space equation containing a product of two (potentially different) sets of structure constants, associated with colour and / or kinematic degrees of freedom. We have considered two generalisations of ref. [83], namely the use of Moyal deformations of the kinematic sector, and also the use of a full two-dimensional diffeomorphism group rather than its more restricted area-preserving counterpart. This gives new kinematic structure constants, and our web of theories - summarised in table 1 - consist of all possible combinations of these various building blocks.

We have also studied the integrability properties of the web of theories. This aspect was not considered in ref. [83], and we discussed how the well-known integrability constructions (e.g., Lax pairs) in self-dual Yang-Mills theory and self-dual gravity are related in a manner expected by the double copy. More generally, for the web of theories summarised in table 1 , the only integrable theories are those for which at least one of the two sets of structure constants is $X\left(p_{1}, p_{2}\right)$, associated to sdiff, i.e., the Lie algebra of area preserving diffeomorphisms. These include self-dual Yang-Mills theory, self-dual gravity and its single Moyal deformation, and hyper-Hermitian gravity (an extension of self-dual gravity associated to hyper-Hermitian manifolds instead of hyper-Kähler manifolds). In those cases, it is possible to construct a Lax pair, and to relate it to an infinite hierarchy of linearised symmetries, expressed as conserved currents. When none of the two sets of structure constants was associated to sdiff, the integrability construction failed. In these cases, we may still have a presumptive Lax pair interpretation, and a bracket based on a deformation of the standard Lie or Poisson brackets, but this bracket fails to obey the Jacobi relation. This agrees with 
previous literature, e.g., ref. [121] regarding Moyal-deformed brackets. We have the interesting consequence that singly Moyal-deformed self-dual gravity can be thought of as "inheriting" its integrability from one of its constituent gauge theories under the double copy, i.e., from the one with kinematic algebra $X\left(p_{1}, p_{2}\right)$ and not the one with kinematic algebra $X^{M}\left(p_{1}, p_{2}\right)$. The picture that emerges is that, when integrability exists, it does so because at least one of the sides of the double copy is integrable. In the cases at hand, all integrable theories are related to self-dual Yang-Mills theory by substitution of $f^{a b c}$ by other structure constants, which can be thought of as arising from a 'symmetry reduction'. Hence, the class of integrable theories considered here is consistent with Ward's conjecture [134, 135].

There are a number of possible avenues for further work. Firstly, it would useful to establish the possible physical interpretation and applications of the theories that have been introduced in order to complete the web obtained via the double copy. This includes the question of whether or not they can be furnished with a geometric interpretation. Secondly, it would be very interesting to determine whether the usual non-commutative deformation of self-dual Yang-Mills theory, discussed briefly in section 3.2, also admits colour-kinematics duality and double copy interpretations, despite the fact that its colour structure is very different from that of the undeformed theory. If such an extension of our approach exists, it would also be interesting to study whether the conclusions would persist under known transformations of the star product (e.g. those of refs. [136, 137], which may to lead to different quantum field theories). Finally, our hope is that the results of our study may stimulate the construction of yet more double copy examples, which may possibly in turn enhance our understanding of conventional Yang-Mills and gravity beyond the self-dual sector.

\section{Acknowledgments}

We are grateful to Maciej Dunajski and Maciej Przanowski for useful discussions. This work has been supported by the U.K. Science and Technology Facilities Council (STFC) Consolidated Grant ST/P000754/1 "String theory, gauge theory and duality", and by the European Union Horizon 2020 research and innovation programme under the Marie Skłodowska-Curie grant agreement No. 764850 "SAGEX". EC is supported by the National Council of Science and Technology (CONACYT). AL is supported by the Department of Energy under Award Number DE-SC0009937. RM is supported by a Royal Society University Research Fellowship.

Open Access. This article is distributed under the terms of the Creative Commons Attribution License (CC-BY 4.0), which permits any use, distribution and reproduction in any medium, provided the original author(s) and source are credited.

\section{References}

[1] H. Kawai, D.C. Lewellen and S.-H. Henry Tye, A relation between tree amplitudes of closed and open strings, Nucl. Phys. B 269 (1986) 1 [InSPIRE].

[2] Z. Bern, J.J.M. Carrasco and H. Johansson, New relations for gauge-theory amplitudes, Phys. Rev. D 78 (2008) 085011 [arXiv:0805.3993] [INSPIRE]. 
[3] Z. Bern, J.J.M. Carrasco and H. Johansson, Perturbative quantum gravity as a double copy of gauge theory, Phys. Rev. Lett. 105 (2010) 061602 [arXiv:1004.0476] [InSPIRE].

[4] Z. Bern, T. Dennen, Y.-T. Huang and M. Kiermaier, Gravity as the square of gauge theory, Phys. Rev. D 82 (2010) 065003 [arXiv: 1004.0693] [InSPIRE].

[5] R. Monteiro, D. O'Connell and C.D. White, Black holes and the double copy, JHEP 12 (2014) 056 [arXiv: 1410.0239] [inSPIRE].

[6] A. Luna, R. Monteiro, D. O'Connell and C.D. White, The classical double copy for Taub-NUT spacetime, Phys. Lett. B $\mathbf{7 5 0}$ (2015) 272 [arXiv:1507.01869] [INSPIRE].

[7] A. Luna, R. Monteiro, I. Nicholson, D. O'Connell and C.D. White, The double copy: Bremsstrahlung and accelerating black holes, JHEP 06 (2016) 023 [arXiv: 1603.05737] [INSPIRE].

[8] M. Carrillo-González, R. Penco and M. Trodden, The classical double copy in maximally symmetric spacetimes, JHEP 04 (2018) 028 [arXiv:1711.01296] [INSPIRE].

[9] N. Bahjat-Abbas, A. Luna and C.D. White, The Kerr-Schild double copy in curved spacetime, JHEP 12 (2017) 004 [arXiv:1710.01953] [INSPIRE].

[10] D.S. Berman, E. Chacón, A. Luna and C.D. White, The self-dual classical double copy, and the Eguchi-Hanson instanton, JHEP 01 (2019) 107 [arXiv: 1809.04063] [INSPIRE].

[11] I. Bah, R. Dempsey and P. Weck, Kerr-Schild double copy and complex worldlines, JHEP 02 (2020) 180 [arXiv: 1910.04197] [INSPIRE].

[12] M. Carrillo González, B. Melcher, K. Ratliff, S. Watson and C.D. White, The classical double copy in three spacetime dimensions, JHEP 07 (2019) 167 [arXiv:1904.11001] [INSPIRE].

[13] A. Banerjee, E.O. Colgáin, J.A. Rosabal and H. Yavartanoo, Ehlers as EM duality in the double copy, Phys. Rev. D 102 (2020) 126017 [arXiv:1912.02597] [INSPIRE].

[14] A. Ilderton, Screw-symmetric gravitational waves: a double copy of the vortex, Phys. Lett. B 782 (2018) 22 [arXiv:1804.07290] [INSPIRE].

[15] R. Monteiro, I. Nicholson and D. O'Connell, Spinor-helicity and the algebraic classification of higher-dimensional spacetimes, Class. Quant. Grav. 36 (2019) 065006 [arXiv: 1809.03906] [INSPIRE].

[16] A. Luna, R. Monteiro, I. Nicholson and D. O'Connell, Type D spacetimes and the Weyl double copy, Class. Quant. Grav. 36 (2019) 065003 [arXiv:1810.08183] [inSPIRE].

[17] K. Lee, Kerr-Schild double field theory and classical double copy, JHEP 10 (2018) 027 [arXiv: 1807.08443] [INSPIRE].

[18] W. Cho and K. Lee, Heterotic Kerr-Schild double field theory and classical double copy, JHEP 07 (2019) 030 [arXiv: 1904.11650] [inSPIRE].

[19] K. Kim, K. Lee, R. Monteiro, I. Nicholson and D. Peinador Veiga, The classical double copy of a point charge, JHEP 02 (2020) 046 [arXiv: 1912.02177] [INSPIRE].

[20] L. Alfonsi, C.D. White and S. Wikeley, Topology and Wilson lines: global aspects of the double copy, JHEP 07 (2020) 091 [arXiv:2004.07181] [INSPIRE].

[21] N. Bahjat-Abbas, R. Stark-Muchão and C.D. White, Monopoles, shockwaves and the classical double copy, JHEP 04 (2020) 102 [arXiv:2001.09918] [INSPIRE]. 
[22] C.D. White, Exact solutions for the biadjoint scalar field, Phys. Lett. B 763 (2016) 365 [arXiv: 1606.04724] [INSPIRE].

[23] P.-J. De Smet and C.D. White, Extended solutions for the biadjoint scalar field, Phys. Lett. B 775 (2017) 163 [arXiv: 1708.01103] [INSPIRE].

[24] N. Bahjat-Abbas, R. Stark-Muchão and C.D. White, Biadjoint wires, Phys. Lett. B 788 (2019) 274 [arXiv: 1810.08118] [INSPIRE].

[25] G. Elor, K. Farnsworth, M.L. Graesser and G. Herczeg, The Newman-Penrose map and the classical double copy, JHEP 12 (2020) 121 [arXiv: 2006. 08630] [INSPIRE].

[26] M.K. Gumus and G. Alkac, More on the classical double copy in three spacetime dimensions, Phys. Rev. D 102 (2020) 024074 [arXiv: 2006.00552] [INSPIRE].

[27] C. Keeler, T. Manton and N. Monga, From Navier-Stokes to Maxwell via Einstein, JHEP 08 (2020) 147 [arXiv: 2005. 04242] [inSPIRE].

[28] N. Arkani-Hamed, Y.-T. Huang and D. O'Connell, Kerr black holes as elementary particles, JHEP 01 (2020) 046 [arXiv:1906.10100] [INSPIRE].

[29] Y.-T. Huang, U. Kol and D. O'Connell, Double copy of electric-magnetic duality, Phys. Rev. D 102 (2020) 046005 [arXiv: 1911.06318] [INSPIRE].

[30] R. Alawadhi, D.S. Berman, B. Spence and D. Peinador Veiga, S-duality and the double copy, JHEP 03 (2020) 059 [arXiv: 1911.06797] [INSPIRE].

[31] N. Moynihan, Kerr-Newman from minimal coupling, JHEP 01 (2020) 014 [arXiv: 1909.05217] [INSPIRE].

[32] R. Alawadhi, D.S. Berman and B. Spence, Weyl doubling, JHEP 09 (2020) 127 [arXiv: 2007.03264] [INSPIRE].

[33] D.A. Easson, C. Keeler and T. Manton, Classical double copy of nonsingular black holes, Phys. Rev. D 102 (2020) 086015 [arXiv:2007. 16186] [inSPIRE].

[34] E. Casali and A. Puhm, Double copy for celestial amplitudes, Phys. Rev. Lett. 126 (2021) 101602 [arXiv:2007.15027] [INSPIRE].

[35] A. Cristofoli, Gravitational shock waves and scattering amplitudes, JHEP 11 (2020) 160 [arXiv: 2006. 08283] [INSPIRE].

[36] A. Luna et al., Perturbative spacetimes from Yang-Mills theory, JHEP 04 (2017) 069 [arXiv: 1611.07508] [INSPIRE].

[37] W.D. Goldberger and A.K. Ridgway, Radiation and the classical double copy for color charges, Phys. Rev. D 95 (2017) 125010 [arXiv:1611.03493] [INSPIRE].

[38] W.D. Goldberger, S.G. Prabhu and J.O. Thompson, Classical gluon and graviton radiation from the bi-adjoint scalar double copy, Phys. Rev. D 96 (2017) 065009 [arXiv:1705.09263] [INSPIRE].

[39] W.D. Goldberger and A.K. Ridgway, Bound states and the classical double copy, Phys. Rev. D 97 (2018) 085019 [arXiv: 1711.09493] [INSPIRE].

[40] W.D. Goldberger, J. Li and S.G. Prabhu, Spinning particles, axion radiation, and the classical double copy, Phys. Rev. D 97 (2018) 105018 [arXiv:1712.09250] [INSPIRE].

[41] C.-H. Shen, Gravitational radiation from color-kinematics duality, JHEP 11 (2018) 162 [arXiv: 1806. 07388] [INSPIRE]. 
[42] M. Carrillo González, R. Penco and M. Trodden, Radiation of scalar modes and the classical double copy, JHEP 11 (2018) 065 [arXiv: 1809.04611] [INSPIRE].

[43] J. Plefka, J. Steinhoff and W. Wormsbecher, Effective action of dilaton gravity as the classical double copy of Yang-Mills theory, Phys. Rev. D 99 (2019) 024021 [arXiv: 1807.09859] [INSPIRE].

[44] J. Plefka, C. Shi, J. Steinhoff and T. Wang, Breakdown of the classical double copy for the effective action of dilaton-gravity at NNLO, Phys. Rev. D 100 (2019) 086006 [arXiv: 1906.05875] [INSPIRE].

[45] W.D. Goldberger and J. Li, Strings, extended objects, and the classical double copy, JHEP 02 (2020) 092 [arXiv: 1912.01650] [INSPIRE].

[46] A.P.V. and A. Manu, Classical double copy from color kinematics duality: a proof in the soft limit, Phys. Rev. D 101 (2020) 046014 [arXiv:1907.10021] [INSPIRE].

[47] A. Anastasiou, L. Borsten, M.J. Duff, L.J. Hughes and S. Nagy, Yang-Mills origin of gravitational symmetries, Phys. Rev. Lett. 113 (2014) 231606 [arXiv:1408.4434] [INSPIRE].

[48] L. Borsten and M.J. Duff, Gravity as the square of Yang-Mills?, Phys. Scripta 90 (2015) 108012 [arXiv: 1602.08267] [INSPIRE].

[49] A. Anastasiou et al., Twin supergravities from Yang-Mills theory squared, Phys. Rev. D 96 (2017) 026013 [arXiv: 1610.07192] [inSPIRE].

[50] G.L. Cardoso, S. Nagy and S. Nampuri, A double copy for $N=2$ supergravity: a linearised tale told on-shell, JHEP 10 (2016) 127 [arXiv:1609.05022] [INSPIRE].

[51] L. Borsten, $D=6, N=(2,0)$ and $N=(4,0)$ theories, Phys. Rev. D 97 (2018) 066014 [arXiv: 1708.02573] [INSPIRE].

[52] A. Anastasiou, L. Borsten, M.J. Duff, A. Marrani, S. Nagy and M. Zoccali, The mile high magic pyramid, Contemp. Math. 721 (2019) 1 [arXiv:1711.08476] [INSPIRE].

[53] A. Anastasiou, L. Borsten, M.J. Duff, S. Nagy and M. Zoccali, Gravity as gauge theory squared: a ghost story, Phys. Rev. Lett. 121 (2018) 211601 [arXiv: 1807.02486] [INSPIRE].

[54] G. Lopes Cardoso, G. Inverso, S. Nagy and S. Nampuri, Comments on the double copy construction for gravitational theories, PoS (CORFU2017) 177 (2018) [arXiv:1803.07670] [INSPIRE].

[55] A. Luna, S. Nagy and C. White, The convolutional double copy: a case study with a point, JHEP 09 (2020) 062 [arXiv:2004.11254] [INSPIRE].

[56] L. Borsten and S. Nagy, The pure BRST Einstein-Hilbert Lagrangian from the double-copy to cubic order, JHEP 07 (2020) 093 [arXiv: 2004.14945] [INSPIRE].

[57] L. Borsten, B. Jurčo, H. Kim, T. Macrelli, C. Sämann and M. Wolf, BRST-Lagrangian double copy of Yang-Mills theory, arXiv:2007.13803 [INSPIRE].

[58] A. Luna, I. Nicholson, D. O'Connell and C.D. White, Inelastic black hole scattering from charged scalar amplitudes, JHEP 03 (2018) 044 [arXiv:1711.03901] [INSPIRE].

[59] D.A. Kosower, B. Maybee and D. O'Connell, Amplitudes, observables, and classical scattering, JHEP 02 (2019) 137 [arXiv:1811.10950] [INSPIRE].

[60] B. Maybee, D. O'Connell and J. Vines, Observables and amplitudes for spinning particles and black holes, JHEP 12 (2019) 156 [arXiv:1906.09260] [INSPIRE]. 
[61] Y.F. Bautista and A. Guevara, On the double copy for spinning matter, arXiv:1908.11349 [INSPIRE].

[62] H. Johansson and A. Ochirov, Double copy for massive quantum particles with spin, JHEP 09 (2019) 040 [arXiv: 1906.12292] [INSPIRE].

[63] Y.F. Bautista and A. Guevara, From scattering amplitudes to classical physics: universality, double copy and soft theorems, arXiv:1903.12419 [INSPIRE].

[64] C. Cheung, I.Z. Rothstein and M.P. Solon, From scattering amplitudes to classical potentials in the post-Minkowskian expansion, Phys. Rev. Lett. 121 (2018) 251101 [arXiv: 1808.02489] [INSPIRE].

[65] Z. Bern, C. Cheung, R. Roiban, C.-H. Shen, M.P. Solon and M. Zeng, Black hole binary dynamics from the double copy and effective theory, JHEP 10 (2019) 206 [arXiv: 1908.01493] [INSPIRE].

[66] Z. Bern, C. Cheung, R. Roiban, C.-H. Shen, M.P. Solon and M. Zeng, Scattering amplitudes and the conservative hamiltonian for binary systems at third post-Minkowskian order, Phys. Rev. Lett. 122 (2019) 201603 [arXiv:1901.04424] [INSPIRE].

[67] Z. Bern, A. Luna, R. Roiban, C.-H. Shen and M. Zeng, Spinning black hole binary dynamics, scattering amplitudes and effective field theory, arXiv:2005.03071 [INSPIRE].

[68] G. Kälin and R.A. Porto, From boundary data to bound states, JHEP 01 (2020) 072 [arXiv: 1910.03008] [InSPIRE].

[69] G. Kälin and R.A. Porto, Post-Minkowskian effective field theory for conservative binary dynamics, JHEP 11 (2020) 106 [arXiv: 2006.01184] [INSPIRE].

[70] G. Kälin, Z. Liu and R.A. Porto, Conservative dynamics of binary systems to third post-Minkowskian order from the effective field theory approach, Phys. Rev. Lett. 125 (2020) 261103 [arXiv: 2007.04977] [INSPIRE].

[71] G.L. Almeida, S. Foffa and R. Sturani, Classical gravitational self-energy from double copy, JHEP 11 (2020) 165 [arXiv: 2008.06195] [InSPIRE].

[72] F. Cachazo, S. He and E.Y. Yuan, Scattering equations and matrices: from Einstein to Yang-Mills, DBI and NLSM, JHEP 07 (2015) 149 [arXiv:1412.3479] [INSPIRE].

[73] H. Johansson and A. Ochirov, Pure gravities via color-kinematics duality for fundamental matter, JHEP 11 (2015) 046 [arXiv:1407.4772] [INSPIRE].

[74] H. Johansson and A. Ochirov, Color-kinematics duality for QCD amplitudes, JHEP 01 (2016) 170 [arXiv: 1507.00332] [INSPIRE].

[75] M. Chiodaroli, M. Günaydin, H. Johansson and R. Roiban, Spontaneously broken Yang-Mills-Einstein supergravities as double copies, JHEP 06 (2017) 064 [arXiv: 1511.01740] [INSPIRE].

[76] M. Chiodaroli, M. Günaydin, H. Johansson and R. Roiban, Complete construction of magical, symmetric and homogeneous $N=2$ supergravities as double copies of gauge theories, Phys. Rev. Lett. 117 (2016) 011603 [arXiv: 1512.09130] [INSPIRE].

[77] J.J.M. Carrasco, C.R. Mafra and O. Schlotterer, Abelian Z-theory: NLSM amplitudes and $\alpha^{\prime}$-corrections from the open string, JHEP 06 (2017) 093 [arXiv:1608.02569] [INSPIRE].

[78] J.J.M. Carrasco, C.R. Mafra and O. Schlotterer, Semi-abelian Z-theory: NLSM+ $\phi^{3}$ from the open string, JHEP 08 (2017) 135 [arXiv:1612.06446] [INSPIRE]. 
[79] A. Anastasiou, L. Borsten, M.J. Duff, A. Marrani, S. Nagy and M. Zoccali, Are all supergravity theories Yang-Mills squared?, Nucl. Phys. B 934 (2018) 606 [arXiv: 1707.03234] [INSPIRE].

[80] T. Azevedo, M. Chiodaroli, H. Johansson and O. Schlotterer, Heterotic and bosonic string amplitudes via field theory, JHEP 10 (2018) 012 [arXiv:1803.05452] [INSPIRE].

[81] Z. Bern, J.J. Carrasco, M. Chiodaroli, H. Johansson and R. Roiban, The duality between color and kinematics and its applications, arXiv:1909.01358 [INSPIRE].

[82] L. Borsten, Gravity as the square of gauge theory: a review, Riv. Nuovo Cim. 43 (2020) 97 [INSPIRE].

[83] R. Monteiro and D. O'Connell, The kinematic algebra from the self-dual sector, JHEP 07 (2011) 007 [arXiv: 1105.2565] [INSPIRE].

[84] A. Parkes, A cubic action for selfdual Yang-Mills, Phys. Lett. B 286 (1992) 265 [hep-th/9203074] [INSPIRE].

[85] J.F. Plebański, Some solutions of complex Einstein equations, J. Math. Phys. 16 (1975) 2395 [INSPIRE].

[86] N.E.J. Bjerrum-Bohr, P.H. Damgaard, T. Sondergaard and P. Vanhove, Monodromy and Jacobi-like relations for color-ordered amplitudes, JHEP 06 (2010) 003 [arXiv:1003.2403] [INSPIRE].

[87] C.R. Mafra, O. Schlotterer and S. Stieberger, Explicit BCJ numerators from pure spinors, JHEP 07 (2011) 092 [arXiv: 1104.5224] [INSPIRE].

[88] N.E.J. Bjerrum-Bohr, P.H. Damgaard, R. Monteiro and D. O'Connell, Algebras for amplitudes, JHEP 06 (2012) 061 [arXiv:1203.0944] [INSPIRE].

[89] C.-H. Fu, Y.-J. Du and B. Feng, An algebraic approach to BCJ numerators, JHEP 03 (2013) 050 [arXiv: 1212.6168] [INSPIRE].

[90] R. Monteiro and D. O'Connell, The kinematic algebras from the scattering equations, JHEP 03 (2014) 110 [arXiv:1311.1151] [INSPIRE].

[91] C.R. Mafra and O. Schlotterer, Multiparticle SYM equations of motion and pure spinor BRST blocks, JHEP 07 (2014) 153 [arXiv:1404.4986] [INSPIRE].

[92] S.G. Naculich, Scattering equations and virtuous kinematic numerators and dual-trace functions, JHEP 07 (2014) 143 [arXiv:1404.7141] [INSPIRE].

[93] C.R. Mafra and O. Schlotterer, Berends-Giele recursions and the BCJ duality in superspace and components, JHEP 03 (2016) 097 [arXiv: 1510.08846] [INSPIRE].

[94] N.E.J. Bjerrum-Bohr, J.L. Bourjaily, P.H. Damgaard and B. Feng, Manifesting color-kinematics duality in the scattering equation formalism, JHEP 09 (2016) 094 [arXiv: 1608.00006] [INSPIRE].

[95] Y.-J. Du and F. Teng, BCJ numerators from reduced Pfaffian, JHEP 04 (2017) 033 [arXiv: 1703.05717] [INSPIRE].

[96] A. Edison and F. Teng, Efficient calculation of crossing symmetric BCJ tree numerators, JHEP 12 (2020) 138 [arXiv: 2005. 03638] [INSPIRE].

[97] C. Cheung and C.-H. Shen, Symmetry for flavor-kinematics duality from an action, Phys. Rev. Lett. 118 (2017) 121601 [arXiv:1612.00868] [INSPIRE]. 
[98] C.-H. Fu and K. Krasnov, Colour-kinematics duality and the Drinfeld double of the Lie algebra of diffeomorphisms, JHEP 01 (2017) 075 [arXiv: 1603.02033] [INSPIRE].

[99] G. Chen, H. Johansson, F. Teng and T. Wang, On the kinematic algebra for BCJ numerators beyond the MHV sector, JHEP 11 (2019) 055 [arXiv: 1906.10683] [INSPIRE].

[100] S. Mizera, Kinematic Jacobi identity is a residue theorem: geometry of color-kinematics duality for gauge and gravity amplitudes, Phys. Rev. Lett. 124 (2020) 141601 [arXiv: 1912.03397] [INSPIRE].

[101] H. Frost and L. Mason, Lie polynomials and a twistorial correspondence for amplitudes, arXiv: 1912.04198 [INSPIRE].

[102] J.E. Moyal, Quantum mechanics as a statistical theory, Proc. Cambridge Phil. Soc. 45 (1949) 99 [inSPIRE].

[103] H.J. Groenewold, On the principles of elementary quantum mechanics, Physica 12 (1946) 405 [INSPIRE].

[104] G.A. Baker, Formulation of quantum mechanics based on the quasi-probability distribution induced on phase space, Phys. Rev. 109 (1958) 2198 [INSPIRE].

[105] C.K. Zachos, D.B. Fairlie and T.L. Curtright eds., Quantum mechanics in phase space, World Scientific, Singapore (2005) [INSPIRE].

[106] M. Li and Y. Wu, Physics in non-commutative world: field theories. I, Rinton Press, U.S.A. (2002).

[107] P. Fletcher, The uniqueness of the Moyal algebra, Phys. Lett. B 248 (1990) 323 [InSPIRE].

[108] J.F. Plebański, M. Przanowski, B. Rajca and J. Tosiek, The Moyal deformation of the second heavenly equation, Acta Phys. Polon. B 26 (1995) 889 [INSPIRE].

[109] J.F. Plebański, M. Przanowski and H. García-Compean, From selfdual Yang-Mills fields to selfdual gravity, Acta Phys. Polon. B 25 (1994) 1079 [inSPIRE].

[110] Q.-H. Park, 2D sigma model approach to $4 D$ instantons, Int. J. Mod. Phys. A 7 (1992) 1415 [INSPIRE].

[111] E. Chacón and H. García-Compeán, Self-dual gravity via Hitchin's equations, J. Math. Phys. 60 (2019) 052502 [arXiv: 1812.08962] [INSPIRE].

[112] M. Dunajski, L.J. Mason and N.M.J. Woodhouse, From $2 d$ integrable systems to self-dual gravity, J. Phys. A 31 (1998) 6019.

[113] J.F. Plebański, M. Przanowski and H. García-Compeán, From principal chiral model to selfdual gravity, Mod. Phys. Lett. A 11 (1996) 663 [hep-th/9509092] [INSPIRE].

[114] Q.-H. Park, Selfdual gravity as a large $N$ limit of the two-dimensional nonlinear $\sigma$ model, Phys. Lett. B 238 (1990) 287 [InSPIRE].

[115] L.J. Mason and E.T. Newman, A connection between the Einstein and Yang-Mills equations, Commun. Math. Phys. 121 (1989) 659 [INSPIRE].

[116] M. Dunajski, The twisted photon associated to hyper-Hermitian four manifolds, J. Geom. Phys. 30 (1999) 266 [math.DG/9808137] [INSPIRE].

[117] M. Dunajski, Solitons, instantons and twistors, Oxford University Press, Oxford, U.K. (2010). 
[118] D. Cangemi, Self-dual Yang-Mills theory and one-loop maximally helicity violating multi-gluon amplitudes, Nucl. Phys. B 484 (1997) 521 [hep-th/9605208] [inSPIRE].

[119] J.F. Plebański and M. Przanowski, The Lagrangian of a selfdual gravitational field as a limit of the SDYM Lagrangian, Phys. Lett. A 212 (1996) 22 [hep-th/9605233] [INSPIRE].

[120] K. Takasaki, Non-Abelian KP hierarchy with Moyal algebraic coefficients, J. Geom. Phys. 14 (1994) 332 [hep-th/9305169] [INSPIRE].

[121] I.A.B. Strachan, A geometry for multidimensional integrable systems, J. Geom. Phys. 21 (1997) 255 [hep-th/9604142] [INSPIRE].

[122] I.A.B. Strachan, The Moyal algebra and integrable deformations of the selfdual Einstein equations, Phys. Lett. B 283 (1992) 63 [INSPIRE].

[123] M. Maceda and D. Martínez-Carbajal, A Kähler compatible Moyal deformation of the first heavenly equation, SIGMA 15 (2019) 073 [arXiv: 1904.09323] [INSPIRE].

[124] D.B. Fairlie, P. Fletcher and C.K. Zachos, Infinite-dimensional algebras and a trigonometric basis for the classical Lie algebras, J. Math. Phys. 31 (1990) 1088.

[125] S. Formański and M. Przanowski, *-SDYM fields and heavenly spaces: I. *-SDYM equations as an integrable system, J. Phys. A 38 (2005) 4399.

[126] S. Formański and M. Przanowski, *-SDYM fields and heavenly spaces: II. Reductions of the *-SDYM system, J. Phys. A 38 (2005) 9371.

[127] M. Przanowski, S. Formański and F.J. Turrubiates, Nonlinear graviton as a limit of $\operatorname{SL}(N, C)$ chiral fields as $N \rightarrow \infty$, Mod. Phys. Lett. A 13 (1998) 3193 [gr-qc/9905078] [INSPIRE].

[128] K. Takasaki, Anti-selfdual Yang-Mills equations on noncommutative space-time, J. Geom. Phys. 37 (2001) 291 [hep-th/0005194] [INSPIRE].

[129] M. Hamanaka, Noncommutative Ward's conjecture and integrable systems, Nucl. Phys. B 741 (2006) 368 [hep-th/0601209] [inSPIRE].

[130] J.D.E. Grant and I.A.B. Strachan, Hypercomplex integrable systems, Nonlinearity 12 (1999) 1247 [solv-int/9808019] [INSPIRE].

[131] J.D. Finley and J.F. Plebański, Further heavenly metrics and their symmetries, J. Math. Phys. 17 (1976) 585.

[132] M. Dunajski, J.D.E. Grant and I.A.B. Strachan, Multidimensional integrable systems and deformations of Lie algebra homomorphisms, J. Math. Phys. 48 (2007) 093502.

[133] V. Ovsienko and C. Roger, Deforming the Lie algebra of vector fields on $S^{1}$ inside the Poisson algebra on ${ }^{*} T^{*} S^{1}$, Commun. Math. Phys. 198 (1998) 97.

[134] R. Ward, Integrable and solvable systems, and relations among them, Phil. Trans. Roy. Soc. Lond. A 315 (1985) 451.

[135] L. Mason and N. Woodhouse, Integrability, selfduality and twistor theory, Clarendon, Oxford, U.K. (1991).

[136] D. Vassilevich, Is covariant star product unique?, PoS (CNCFG2010) 006 (2010) [arXiv: 1101.4642] [INSPIRE].

[137] B. Jurčo and P. Schupp, Noncommutative Yang-Mills from equivalence of star products, Eur. Phys. J. C 14 (2000) 367 [hep-th/0001032] [INSPIRE]. 